\title{
A Multi-wavelength study of the Pulsar PSR B1929+10 and its X-ray trail
}

\author{
Werner Becker ${ }^{1}$, Michael Kramer ${ }^{2}$, Axel Jessner ${ }^{3}$, Ronald E. Taam ${ }^{4}$, \\ Jian J. Jia ${ }^{5}$, Kwong S. Cheng ${ }^{5}$, Roberto Mignani ${ }^{6}$, Alberto Pellizzoni ${ }^{7}$, \\ Andrea de Luca ${ }^{7}$, Agnieszka Slowikowska ${ }^{8}$, Patrizia Caraveo $^{7}$
}

\begin{abstract}
We report on the emission properties of PSR B1929+10 and its putative trail from a multi-wavelength study performed with XMM-Newton, the ESO New Technology Telescope (NTT) in La Silla (Chile), the Hubble Space Telescope, the Effelsberg 100m Radio Telescope and the Jodrell Bank Radio Observatory. The XMM-Newton observations confirm the existence of the diffuse emission with a trail morphology lying in a direction opposite to the transverse motion of the pulsar. The trail has a length of $\sim 15$ arcmin. Its spectrum is non-thermal and produced by electron-synchrotron emission in the shock between the pulsar wind and the surrounding medium. The emission from the brightest portion of the trail corresponds to $\sim 2 \times 10^{-4}$ of the pulsar's spin-down energy. At a distance of 2.5 arcmin from the pulsar $\sim 4-7 \times 10^{-5} \dot{E}$ is still emitted. Assuming that the electron lifetime against synchrotron cooling is comparable to the source transit time over the X-ray trail length, the magnetic field strength in the trail emitting region is inferred to be $\sim 5 \mu \mathrm{G}$. Optical observations performed in the $\mathrm{H}_{\alpha}$ band did not find the counterpart of the diffuse X-ray trail emission near to the pulsar. Inspecting data from the Effelsberg $11 \mathrm{~cm}$ radio continuum survey of the Galactic plane we discovered an elongated feature apparently coincident with the
\end{abstract}

\footnotetext{
${ }^{1}$ Max-Planck Institut für Extraterrestrische Physik, 85741 Garching bei München, Germany

${ }^{2}$ University of Manchester, Jodrell Bank Observatory, Macclesfield, Cheshire SK11 9DL, UK

${ }^{3}$ Max-Planck Institut für Radioastronomie, Effelsberg, 53902 Bad Münstereifel, Germany

${ }^{4}$ Northwestern University, Dep. of Physics and Astronomy, 2145 Sheridan Road, Evanston, IL 60208

${ }^{5}$ Department of Physics, University of Hong Kong, Hong Kong, China

${ }^{6}$ ESO, Karl-Schwarzschild Strasse 2, 85740 Garching bei München, Germany

${ }^{7}$ Istituto di Astrofisica Spaziale e Fisica Cosmica, via Bassini 15, I-20133 Milano, Italy

${ }^{8}$ Nicolaus Copernicus Astronomical Center, Rabianska 8, 87-100 Torun, Poland
} 
X-ray trail. The emission properties observed from PSR B1929+10 are found to challenge the picture of the emission properties of old non-recycled rotation powered pulsars which was based on low statistical ROSAT and ASCA data. Both, the temporal and spectral X-ray emission properties of PSR B1929+10 are in excellent agreement with a non-thermal and, thus, magnetospheric radiation dominated emission scenario. The pulsar's X-ray spectrum is best described by a single power law model with a photon index of $2.72_{-0.09}^{+0.12}$. A flux contribution from the thermal emission of heated polar caps of $\sim 7 \%$ is inferred from a best fitting composite Planckian and power law spectral model. A pure thermal emission spectrum consisting of two Planckian spectra is regarded as unlikely. A broken power law spectral model with $E_{\text {break }}=0.83_{-0.03}^{+0.05} \mathrm{keV}$ and the photonindex $\alpha_{1}=1.12_{-0.03}^{+0.02}$ and $\alpha_{2}=2.48_{-0.07}^{+0.08}$ can describe the optical and X-ray data entirely in terms of a non-thermal magnetospheric origin. The X-ray pulse profile observed in the $0.2-10 \mathrm{keV}$ band is found to be markedly different from the broad sinusoidal pulse profile seen in the low statistic ROSAT data. Fitting Gaussians to the X-ray light curve indicates the possible existence of three pulse components. A small narrow pulse, characterized by energies greater than $1 \mathrm{keV}$, is found to lead the radio main pulse by $\sim 20^{\circ}$. The fraction of pulsed photons in the $0.2-10 \mathrm{keV}$ band is $32 \pm 4 \%$. For the sub-bands $0.2-1.0 \mathrm{keV}$ and $1.0-2.1$ $\mathrm{keV}$ the pulsed fraction is $24 \pm 5 \%$ and $44 \pm 6 \%$, respectively, indicating a mild energy dependence on a $\sim 2 \sigma$ level. Simulations in the framework of an outergap emission model are able to reproduce the observed X-ray pulse profile and its phase relation relative to the radio pulse.

Subject headings: pulsars:general — pulsars:individual (PSR B1929+10) — stars: neutron — x-ray:stars

\section{INTRODUCTION}

It is fair to say that a consistent scenario for the evolution of the X-ray emission properties of aging rotation-powered pulsars does not yet exist. This surprising fact is largely due to the lack of sufficient observational data. Young and middle aged neutron stars, which emit strong pulsed non-thermal and/or surface hot-spot plus cooling emission, were studied reasonably well by the X-ray observatories EINSTEIN, ROSAT, ASCA and BeppoSAX. In contrast, most old radio pulsars were too faint for a detailed examination of their X-ray emis-

sion by these instruments (cf. Sun et al. 1993; Manning \& Willmore 1994; Becker \& Trümper 1997; Saito 1998). However, especially old (in standards of X-ray detected pulsars) rotation- 
powered non-recycled pulsars are of particular interest for the study of particle acceleration and high energy radiation processes near the neutron star's surface and in its magnetosphere. This is because their ages are intermediate between those of the well-studied young and cooling neutron stars, whose surface may produce copious thermal X-ray photons, and those of very old recycled millisecond pulsars, in which thermal hot-spot and non-thermal magnetospheric X-ray production mechanisms are believed to dominate. Old, non-recycled pulsars (outside globular clusters) therefore aid in answering questions such as "How do the emission properties of the younger pulsars, like Geminga, PSR B0656+14 and PSR B105552 , change as they age from $\sim 10^{5}$ to $10^{7}$ years? Will the thermal emission simply fade away due to cooling with increasing age or will the star be kept hot (at about $0.5-1 \times 10^{5} \mathrm{~K}$ ) over millions of years due to energy dissipation by processes such as internal frictional heating ( $\dot{E}_{\text {diss }} \sim 10^{28}-10^{30} \mathrm{erg} / \mathrm{s}$ ) and crust cracking, as proposed by vortex creeping and pinning models? What happens to the non-thermal, hard-tail emission seen in the X-ray spectra of the middle-aged field pulsars? (See e.g. Saito 1988; Becker \& Aschenbach 2002; De Luca et al. 2005). Will this emission become the dominant source or will this component also decay with time and will only thermal emission from the hot and heated polar-caps remain?"

In order to address these questions, we initiated a program to study the X-ray emission properties of old rotation-powered pulsars with XMM-Newton, aiming to probe and identify the origin of their X-ray radiation. First results from parts of this project have been presented recently, reporting on the pulsars B0950+08, B0823+26, J2043+2740 (Becker et al. 2004) and PSR B0628-28 (Becker et al. 2005) all of which have a spin-down age in the range from about one million to seventeen million years.

The results have been unexpected. If one extrapolates the X-ray emission properties of young and cooling neutron stars to this age bracket, one may expect that the cooling emission fades away and the neutron stars become unobservable at X-rays. It was unforeseen, however, to find the X-ray emission of these old pulsars largely dominated by non-thermal radiation processes. None of the pulsars' X-ray spectra required the addition of a thermal component, consisting of hot polar cap emission, to model their energy spectra. Additional support for an emission scenario dominated by a non-thermal component is given by the observed temporal emission properties. The pulse profiles of PSRs B0950+08 and B0628-28 are not broad and sinusoidal as would have been expected for spin-modulated thermal X-ray emission from heated polar caps, but are double peaked with narrow pulse components and pulsed fractions in the range of $\sim 30-50 \%$.

Nevertheless, these results may not really be in disagreement with some theoretical emission models. Harding \& Muslimov (2001; 2002; 2003) predicted in the framework of their revised space-charge-limited flow model that polar cap heating, as a fraction of the 
spin-down luminosity, increases with pulsar age and should be most efficient for pulsars of spin-down age $\tau \sim 10^{7} \mathrm{yrs}$, if they are in fact producing pairs from curvature radiation photons. However, according to their model, B0950+08 and B0823+26 cannot produce pairs from curvature radiation of primary electrons since they both lie below the curvature radiation pair death line in the $P-\dot{P}$ diagram of radio pulsars (cf. Becker et al. 2004 for a more detailed discussion).

A good candidate object to test these models is PSR B1929+10 which was considered to be prototypical of an old pulsar according to its X-ray emission properties. With a pulse period of $P=226.5 \mathrm{~ms}$ and a period derivative of $\dot{P}=1.16 \times 10^{-15}$, its characteristic age is determined to be $\sim 3 \times 10^{6}$ years. These spin parameters imply a spin-down luminosity of $\log \dot{E}=33.59 \mathrm{erg} \mathrm{s}^{-1}$ and a magnetic field at the neutron star magnetic poles of $B_{\perp} \sim 5 \times 10^{11}$ G. With a radio dispersion measure of $3.176 \mathrm{pc} \mathrm{cm}^{-3}$, the NE2001 Galactic free electron density model of Cordes \& Lazio (2002) predicts a distance of 170 pc. The most recent and most accurate astrometric measurements by Chatterjee et al. (2004), however, result into a precise proper motion determination of $\mu_{\mathrm{RA}}=17.00 \pm 0.27 \mathrm{mas} \mathrm{yr}^{-1}$ and $\mu_{\mathrm{DEC}}=-9.48 \pm 0.37$ mas $\mathrm{yr}^{-1}$ and a parallax that translates into an accurate distance measurement of $d=361_{-8}^{+10}$ pc and a transverse speed of $V_{\perp}=177_{-5}^{+4} \mathrm{~km} \mathrm{~s}^{-1}$.

Thus, PSR B1929+10 is among the closest pulsars known. In addition to its relatively young age it appears to be the brightest among all old non-recycled X-ray detected rotationpowered pulsars. Its X-ray emission was discovered with the EINSTEIN observatory by Helfand (1983). A somewhat more detailed study of its high energy emission properties became possible about ten years later with ROSAT. With about 420 source counts which were recorded with the ROSAT PSPC during a $\sim 45$ ksec exposure Yancopoulos et al. (1994) detected its X-ray emission to be pulsed. The pulse profile was found to be very broad with a single pulse stretching across the entire phase cycle, markedly different from the sharp peak observed in the radio band. The fraction of pulsed photons in the $0.1-2.4 \mathrm{keV}$ band was determined to be $\sim 30 \%$. The limited photon statistics and small bandwidth of the ROSAT detector allowed to fit the pulsar's X-ray spectrum equally well with a blackbody spectrum (representing thermal polar-cap emission, $T \sim 3.2 \times 10^{6} \mathrm{~K}, R_{b b} \sim 20-50 \mathrm{~m}$ ) or with a power law model and a photon-index in the $1 \sigma$ confidence range corresponding to $\alpha \sim 2-2.6$ (Becker \& Trümper 1997). The ROSAT data thus were not able to constrain the nature of the pulsar emission. Similar results were obtained in the analysis of ASCA spectral/timing data by Wang and Halpern (1997) and Saito (1998). The broad sinusoidal pulse profile together with the higher column absorption of $\sim(0.6-1.1) \times 10^{21} \mathrm{~cm}^{-2}$ deduced from power law fits $^{9}$ were taken as indirect arguments and strong indicator for a thermal

\footnotetext{
${ }^{9}$ in comparison to $\sim(1-3) \times 10^{20} \mathrm{~cm}^{-2}$ as obtained from blackbody fits and in apparently better agreement
} 
polar-cap origin of the X-rays.

In contrast, Slowikowska et al. (2005) recently found that a single blackbody spectral model cannot describe the pulsar spectrum if the ROSAT and ASCA observed spectra are modeled in a joint analysis. In their work it is shown that a single power law model or a composite model consisting of either a blackbody plus power law or a two component blackbody spectrum can successfully describe the energy spectrum up to $\sim 7 \mathrm{keV}$. The higher column density fitted by these models is found to be in agreement with that observed for other sources located near to the pulsar's line of sight and at comparable distances (Slowikowska et al. 2005).

Regardless of the nature of its X-radiation, PSR B1929+10 seems to be special for its extended X-ray emission which was discovered by Wang, Li \& Begelman (1993) in their archival study of the Galactic soft X-ray background using deep ROSAT PSPC images. They found that the orientation of the diffuse X-ray emission is almost aligned with the pulsar's proper motion direction, suggesting an interpretation in terms of an X-ray emitting trail behind the pulsar. If indeed associated with the pulsar, the trail could account for $\sim 3 \times 10^{-4}$ of the pulsar's spin-down luminosity although the effective brightness may depend strongly on the density of the ambient interstellar matter. In recent years, confirmation of the trail from a subsequent $\sim 350 \mathrm{ksec}$ deep ROSAT HRI observation has not been reported, casting some doubt on its existence.

Near-UV emission from PSR B1929+10 has been detected in three partly overlapping spectral bands using the Hubble Space Telescope's Faint Object Camera (Pavlov et al. 1996) and the NUV-MAMA detector (Mignani et al. 2002). The pulsar was clearly detected in the U-band $\left(m_{342 W}=25.7\right)$ and with the F130LP $\left(m_{130 L P}=26.9\right)$ and STIS F25QTZ filters, but not in $\mathrm{B}\left(m_{430 W} \geq 26.2\right)$. The nature of the optical emission is uncertain since the paucity of color information makes any spectral fit based on the optical data only merely tentative.

PSR B1929+10 is not only well studied at high energies but has also been extensively observed at radio frequencies. It belongs to the small group of pulsars of which radio emission was detected up to $43 \mathrm{GHz}$ (Kramer et al. 1997). The only other pulsars for which this was possible so far are PSR B0329 + 54, B0355 + 54 and B2021 + 51. The radio spectrum of PSR $\mathrm{B} 1929+10$ is a power law with a photon index of $\alpha=-2.6 \pm 0.04$ in the frequency range $0.4-24 \mathrm{GHz}$ (Maron et al. 2000). Its energy flux at $100 \mathrm{MHz}$ is $950 \mathrm{mJy} \pm 600 \mathrm{mJy}$, but at $43 \mathrm{GHz}$ it is still $0.18 \mathrm{mJy} \pm 0.05 \mathrm{mJy}$ (which is slightly more than extrapolated from the lower frequency data). The main peak of the radio profile, although small compared to the $\mathrm{X}$-ray pulse, has a substructure which can be modeled by six separate components (Kramer 
et al. 1994). Low-level emission connects this main pulse with an interpulse about 180 deg in longitude apart (see e.g. Everett\& Weisberg 2001). The pulsar's viewing geometry has been studied by many authors via the observed polarization angle swing, applying a rotating vector model. Everett \& Weisberg (2001) reviewed the various results and concluded that both, the main and interpulse, are produced by nearly aligned rotation and magnetic axes and are emitted from nearly opposite sides of a wide, hollow cone. They derive an inclination of the magnetic axis with the spin axis of $\alpha \sim 36^{\circ}$ while the impact angle of the line of sight was determined to be $\beta \sim 26^{\circ}$ (cf. Fig.1).

In this paper we report on X-ray, optical H-alpha and radio observations of PSR B1929+10 which were made with XMM-Newton, the ESO New Technology Telescope (NTT) in La Silla (Chile) and the Jodrell Bank Radio Observatory in order to explore the spectral and timing emission properties of this interesting pulsar and its environment. The paper is organized in the following manner: in $\S 2$ we describe the radio, optical H-alpha and XMMNewton observations of PSR B1929+10 and its X-ray trail and provide the details of the data processing and data filtering. The results of the spatial, spectral and timing analysis are given in $§ 3$. A summary and concluding discussion is presented in $\S 4$.

\section{OBSERVATIONS AND DATA REDUCTION}

\subsection{RADIO OBSERVATIONS OF PSR B1929+10}

The ephemerides for the analysis of the X-ray data were obtained from radio observations and the measurement of pulse times-of-arrival (TOAs) using the 76-m Lovell radio telescope at Jodrell Bank Observatory. Table 1 summarizes the radio ephemerides of PSR B1929+10. A dual-channel cryogenic receiver system sensitive to two orthogonal polarizations was used predominantly at frequencies close to $1400 \mathrm{MHz}$. The signals of each polarization were mixed to an intermediate frequency, fed through a multichannel filter-bank and digitized. The data were de-dispersed in hardware and folded on-line according to the pulsar's dispersion measure and topocentric period. The folded pulse profiles were stored for subsequent analysis. In a later off-line processing step, any sub-integrations corrupted by RFI were removed, the polarizations combined and the remaining sub-integrations averaged to produce a single total-intensity profile for the observation. TOAs were subsequently determined by convolving, in the time domain, the averaged profile with a template corresponding to the observing frequency. The uncertainty on the TOA was found using the method, described by Downs \& Reichley (1983) which incorporates the off-pulse RMS noise and the 'sharpness' of the template. These TOAs were transferred to an arrival time at the solar system barycenter using the Jet Propulsion Laboratory DE200 solar system ephemeris (Standish 1982). More details 
can be found in Hobbs et al. (2004). Spectral data from PSR B1929+10 were obtained from the compilation of Maron et al. (2000).

\subsection{OPTICAL OBSERVATIONS OF PSR B1929+10}

We have performed optical $\mathrm{H}_{\alpha}$ and R-band observations of the $4.25 \times 5.41$ arcmin sky region around PSR B1929+10 using the SUperb-Seeing Imager (SuSI2) at the focus of the ESO New Technology Telescope ${ }^{10}$ in La Sila (Chile).

19 exposures of $600 \mathrm{~s}$ each ( $\sim 3.2$ hours total integration time) have been taken on July, 18 and 202004 through the $\mathrm{H}_{\alpha}$ filter (central wavelength $\lambda=6555.28 \stackrel{\circ}{A} ; \Delta \lambda=69.76 \stackrel{\circ}{A}$ ). Additional 15 shorter exposures of $30-60 \mathrm{~s}$ in the R-band filter (central wavelength $\lambda=$ $6415.8 \AA ; \Delta \lambda=1588.9 \AA$ ) ) were taken in order to discriminate $\mathrm{H}_{\alpha}$ line emission from the continuum. In order to compensate for the $8^{\prime \prime}$ gap between the two SUSI2 CCD chips, the exposure sequences were taken with a jitter pattern with typical offset steps of $20^{\prime \prime}$ in RA. All observations have been performed with average airmass of 1.35, clear sky conditions and good seeing $\left(\sim 0.6^{\prime \prime}\right)$. Single exposures were corrected for the instrumental effects (bias and dark subtraction, flat-fielding), cleaned of bad columns and of cosmic rays hits through median filter combination. To account for the uneven exposure map due to the jitter pattern, both the final $\mathrm{H}_{\alpha}$ and R-band images were exposure-corrected.

The image astrometry was recomputed using as a reference the positions of a number of stars selected from the Guide Star Catalogue II (GSC-II), which has an intrinsic absolute astrometric accuracy of $\sim 0$ '.35 per coordinate ${ }^{11}$. The pixel coordinates of the reference stars have been computed by a two-dimensional Gaussian fitting procedure, and transformation from pixel to sky coordinates was then computed using the programme ASTROM ${ }^{12}$, yielding an $\mathrm{rms}$ of $\sim 0$.'08 in both Right Ascension and Declination, which we assume representative of the accuracy of our astrometric solution.

\footnotetext{
${ }^{10}$ See http://www.ls.eso.org/lasilla/sciops/ntt/index.html for a description of the ESO NTT and its instrumentation.

${ }^{11}$ http://www-gsss.stsci.edu/gsc/gsc2/GSC2home.htm

${ }^{12}$ http://star-www.rl.ac.uk/Software/software.htm
} 


\subsection{XMM-NEWTON OBSERVATIONS OF PSR B1929+10}

PSR B1929+10 was observed by XMM-Newton ${ }^{13}$ as part of the EuroPean Imaging Camera (EPIC) guaranteed time program. Because of severe visibility restrictions caused by XMM-Newton's orbit, the sky region in the direction of PSR B1929+10 became accessible for observations of reasonable exposure times only recently. Observations were performed on 2003 November 10 (XMM rev. 718) with a duration of $\sim 11 \mathrm{ksec}$ and five months later on 2004 April 27 (XMM rev. 803) and April 29 (XMM rev. 804) for a duration of $\sim 22 \mathrm{ksec}$ and $\sim 23 \mathrm{ksec}$, respectively. In all three observations the EPIC Positive-Negative charge depleted Silicon Semiconductor (PN camera) was used as the prime instrument. The two Metal Oxid Semiconductor cameras (MOS1 \& MOS2) were operated in PrimeFullWindow mode to obtain imaging and spectral data. The EPIC-PN camera was set up to operate in SmallWindow read-out mode which provides imaging, spectral and timing information with a temporal resolution of $5.67 \mathrm{~ms}$. The higher temporal resolution of the EPIC-PN SmallWindow mode was preferred over other EPIC-PN imaging modes because of its higher temporal resolution, albeit its $\sim 30 \%$ higher dead-time caused a decrease in the net exposure by $\sim 1 / 3$. The medium filter was used for the EPIC-PN and MOS1/2 cameras in all observations to block optical stray light. The expected lower efficiency of the RGS and optical monitor means that they are of limited use for the given exposure time. A summary of exposure times, instrument modes and filters used for the X-ray observations of PSR B1929+10 is given in Table 2 .

XMM-Newton data have been seen to show timing discontinuities in the photon arrival times with positive and negative jumps of the order of one to several seconds (Becker \& Aschenbach 2002; Kirsch et al. 2003). Inspection of the log-files from our processing of raw data reveals that none of the EPIC-PN data taken from PSR B1929+10 exhibit such discontinuities. Nevertheless, for the processing of this PN data, we used a release track version of the XMM-Newton SAS (Standard Analysis Software) for the analysis of the EPIC-PN data. This software detects and corrects most of the timing discontinuities during data processing. In addition, known timing offsets due to ground station and spacecraft clock propagation delays are corrected by this software in using newly reconstructed time correlation (TCX) data. Barycenter correction of the EPIC-PN data and all other analysis steps were performed by using SAS Version 6.1. Data screening for times of high sky background was done by inspecting the light-curves of the EPIC-MOS1/2 and PN data at energies above $10 \mathrm{keV}$. Apart from having a rather high sky background contribution in both April observations,

\footnotetext{
${ }^{13}$ See http://xmm.vilspa.esa.es/ for a description of XMM-Newton, its instrumentation and the various detector modes available for observations.
} 
very strong X-ray emission from soft proton flares is covering about half of these April data sets. The data quality of the shorter November 2003 observation is not reduced by these effects.

In creating the light-curves with bins of $100 \mathrm{~s}$, we rejected those times where the MOS1/2 light-curves had more than 130, 140 and 175 cts/bin in the 2003 November, 2004 April 27

and 29 observations, respectively. For the EPIC-PN data sets, we rejected times with more than 7, 12 and $40 \mathrm{cts} /$ bin. The data screening reduced the effective exposure time for the MOS1/2 and PN-camera to a total of $64 \mathrm{ksec}$ and $23.8 \mathrm{ksec}$, respectively. The net exposure time, after rejecting times of high sky background, are thus only $\sim 57 \%$ and $\sim 43 \%$ of the requested observing time for the MOS1/2 and EPIC-PN detectors.

For the spectral analysis based on the MOS1/2 data we used only those events with a detection pattern between 0 - 12 (i.e. single, double and triple events) and the flag parameter set to less than, or equal to, 1 . The latter criterion excludes events which are located near a hot pixel, or a bright CCD column, or which are near the edge of the CCD. For the EPIC-PN timing and spectral analysis, we used only single and double events, i.e. those which have a pattern parameter of less than, or equal to, 4 and a flag parameter equal to zero. The energy range of the MOS1/2 and EPIC-PN CCDs was restricted to $0.2-10 \mathrm{keV}$ for the spectral and timing analysis.

\section{ANALYSIS OF THE MULTI-WAVELENGTH DATA OF PSR B1929+10}

The X-ray counterpart of PSR B1929+10 is detected with high significance in both the MOS1/2 and EPIC-PN data. The count rates are $0.0283 \pm 0.0001 \mathrm{cts} / \mathrm{s}(\mathrm{MOS} 1 / 2)$ and $0.078 \pm 0.003 \mathrm{cts} / \mathrm{s}(\mathrm{EPIC}-\mathrm{PN})$ within the $0.2-10 \mathrm{keV}$ band. Inspection of the MOS1 $/ 2$ and EPIC-PN images revealed diffuse extended emission at the position of the putative X-ray trail seen in the ROSAT data by Wang, Li and Begelman (1993). Figures 2, 3 and 4 show the images from a re-analysis of the archival ROSAT data and the XMM-Newton MOS1/2 data taken in 2003 November and 2004 April. The angular resolution of the ROSAT detectors is 25 arcsec in the PSPC and 5 arcsec in the HRI while that of XMM-Newton is 15 arcsec (Half Energy Width). As can be seen in Figures 3 and 4, the X-ray trail is clearly detected with high significance and its existence is confirmed by XMM-Newton beyond any doubt.

The length of the trail, as estimated from the PSPC is about 9 arcmin. In the XMMNewton MOS1/2 cameras it is seen down to the edge of the detector which indicates that its length is at least $\sim 15$ arcmin, albeit its significance decreases for larger off-axis angles. Its orientation is along the pulsar's proper motion direction which is indicated by arrows in 
Figures 2 and 3. The width of the trail has a diameter of slightly more than 1 arcmin at a position near to the pulsar. The larger point spread function for regions observed off-axis makes it difficult to estimate the trail size at positions more distant from the pulsar. A re-analysis of the deep archival ROSAT HRI data showed that a small portion of the X-ray trail emission is also present in these data (cf. inset in Figure 2), albeit with a very low significance level because of the much higher background noise of the HRI detector relative to the ROSAT PSPC. Computing the XMM-Newton MOS1/2 count rate of a 1 arcmin circular trail region located behind the pulsar along its proper motion direction, we find $(5.1 \pm 0.9) \times 10^{-3} \mathrm{cts} / \mathrm{s}$. The counting rate in a circle of the same size but located $\sim 2.5$ arcmin distant from the pulsar along the trail is $(1.7 \pm 0.9) \times 10^{-3} \mathrm{cts} / \mathrm{s}$.

In the first half of the 80s the Effelsberg 100-m radio telescope has mapped the entire Galactic Plane in the latitude range $\pm 5^{\circ}$ at $11 \mathrm{~cm}$ wavelength (Reich et al. 1990). Inspecting this data for a possible radio counterpart of the pulsars's X-ray trail we discovered an elongated feature apparently coincident with it. This detection provides supporting, and independent, evidence for the existence of a synchrotron trail behind the pulsar. Figure 5 shows a $\sim 30 \times 30$ arcmin section of the $11 \mathrm{~cm}$ radio map around the pulsar as extracted from the Effelsberg public data archive.

The NTT $\mathrm{H}_{\alpha}$ image which shows the $4.25 \times 5.41$ arcmin sky region around PSR B1929+10 is shown in Figure 6. Contour lines from the MOS1/2 image are overlaid. The inspection of the $\mathrm{H}_{\alpha}$ image and of the ratio of the $\mathrm{H}_{\alpha}$ to $R$-band images does not reveal any diffuse structure that could be related to the brighter parts of the pulsar's X-ray trail. We performed a rough flux calibration of the $\mathrm{H}_{\alpha}$ image by computing the $\mathrm{H}_{\alpha}$ fluxes corresponding to the $\mathrm{R}$ magnitudes of a sample of GSC-II stars which are included in the field assuming blackbody spectra with the appropriate stellar temperatures. We then derived the relation between the instrumental magnitude and the $\mathrm{H}_{\alpha}$ fluxes. A flux upper limit of $\sim 10^{-16} \mathrm{erg} \mathrm{s}^{-1} \mathrm{~cm}^{-2} \operatorname{arcsec}^{-2}$ in the $\mathrm{H}_{\alpha}$ band can be set for the sky region around the pulsar.

As can be seen in Figure 6, there is a bright star near to the position of PSR B1929+10. A second bright star seems to coincide with the brighter part of the trail near to the pulsar. In view of the density of stars in the observed field the chance probability to have a star near to the position of an X-ray source is quite high. We investigated therefore whether these stars contribute to the flux recorded from PSR B1929+10 and the brighter part of its trail. The bright star close to PSR B1929+10 is of K(4-6)III-I type (Kouwenhoven \& van der Berg 2001 ) and has a magnitude in $\mathrm{B}$ and $\mathrm{R}$ of $\sim 14.85$ and $\sim 13.44$, respectively. The bright star within the trail is possibly of K2-4 class and according to the GSC-II and the 2MASS catalogues has B, R, J and K magnitudes of $\sim 13.97, \sim 12.13, \sim 10.6$ and $\sim 10.0$, respectively. With $\log \left(F_{x} / F_{\text {opt }}\right)=2.77 \pm 1.0$ (Krautter et al. 1999), we find for a mekal plasma model 
with $\mathrm{kT}=0.35$, solar abundances, and a column absorption of $N_{H} \sim 10^{21} \mathrm{~cm}^{-2}$ a possible contribution from these stars to the soft channels in XMM-Newton of $\leq 10-20 \%$. Given the very large spread in the emission properties of $\mathrm{K}$ stars and taking its colors into account the star near to PSR B1929+10 is possibly a bright giant, and thus could lie towards the lower end of the $F_{x} / F_{\text {opt }}$ range (e.g. Zickgraf et al. 2005) which then would imply a negligible flux contribution in the pulsar extraction region. As far as the pulsar's X-ray trail is concerned it is clear from its $\geq 15$ arcmin length and from its hard X-ray spectrum (cf. §3.2.2) that this trail is not due to unresolved foreground or background sources.

\subsection{TIMING ANALYSIS}

We used all the EPIC-PN SmallWindow mode data for the timing analysis, including those times of high sky background which were excluded for spatial and spectral analysis. Experience has shown that this does not affect the results from the timing analysis if the sky background is properly taken into account in all pulsed-fraction estimates. The temporal resolution of $5.67 \mathrm{~ms}$, available in the SmallWindow mode, is more than sufficient to resolve the $226 \mathrm{~ms}$ period from PSR B1929+10.

Events were selected from a circle of 20 arcsec radius centered on the pulsar. This extraction region contains $75 \%$ of the point source flux. For the barycenter correction we applied the standard procedures for XMM-Newton data using barycen-1.17.3 and the JPL DE200 Earth ephemeris (Standish 1982) to convert photon arrival times from the spacecraft to the solar system barycenter (SSB) and the barycentric dynamical time (TDB). The pulsar radio timing position (cf. Table 1) was used for the barycenter correction. The spin-parameters $f$ and $\dot{f}$ of PSR B1929+10 are known with high precision from our contemporaneous radio observations, covering all XMM-Newton orbits relevant for our analysis. PSR B1929+10 is not known to show timing irregularities (glitches) so that we can fold the photon arrival times using the pulsar's radio ephemeris. The statistical significance for the presence of a periodic signal was obtained from a $Z_{n}^{2}$-test with $1-10$ harmonics in combination with the H-Test to determine the optimal number of harmonics (De Jager 1987; Buccheri \& De Jager 1989). The optimal number of phase bins for the representation of the pulse profile was determined by taking into account the signal's Fourier power and the optimal number of harmonics deduced from the H-Test (see Becker \& Trümper 1999 and references therein).

Within the $0.2-10 \mathrm{keV}$ energy band, 5736 events were available for the timing analysis of which $\sim 51 \%$ are estimated to be instrument and sky background. The $Z_{n}^{2}$-test gave 82.14 for $n=6$ harmonics $\left(Z_{1}^{2}=48.61\right)$. According to the H-Test, the probability of measuring $Z_{6}^{2}=82.14$ by chance is $\sim 1.6 \times 10^{-12}$. The significance of the pulsed signal thus 
is comparable with that found recently in the old pulsars B0950+08 (Becker et al. 2004) and PSR B0628-28 (Becker et al. 2005).

The $0.2-10 \mathrm{keV}$ pulse profile is shown together with a radio profile observed at 1.4 $\mathrm{GHz}$ in Figure 7. It reveals a significant deviation from a sinusoidal pulse shape. The Xray pulse profile is found to consist of at least two pulse peaks, a broader component of $\sim 273^{\circ}$ width, plus a narrow component of $\sim 44^{\circ}$ width. Taking the center of mass of the pulse as a reference point, the narrow X-ray pulse appears to be slightly phase shifted (by $\sim 22^{\circ}$ ) from the location of the main radio peak, although both components overlap well in phase. The broader pulse appears to have substructure which indicates that this component could consist of two narrower pulse peaks which are not fully resolved by the available data. Indeed, modeling the profile with three Gaussians yields acceptable results, giving support to this interpretation. The fitted functions together with the post-fit residuals are shown in Figure 8. The centers and widths (FWHM) of each of these three components obtained from the fit are, in pulse longitudes, $\left(112^{\circ}, 80^{\circ}\right),\left(220^{\circ}, 75^{\circ}\right)$, and $\left(346^{\circ}, 22^{\circ}\right)$, respectively. The uncertainty in the center position of each component is estimated as $\sim 8^{\circ}(1 \sigma)$.

In order to search for any energy dependence in the pulsar's temporal X-ray emission properties, we restricted the timing analysis to the $0.2-1.0 \mathrm{keV}, 1.0-2.1 \mathrm{keV}$, and $2.1-10$ $\mathrm{keV}$ energy bands. This analysis shows that the pulsed signal appears to be most significant if we only take events which are recorded at energies below $\sim 2.1 \mathrm{keV}$. The maximum $Z_{n^{-}}^{2}$ values found for the pulsed signal in the three energy bands are $Z_{1}^{2}=32.12, Z_{3}^{2}=52.1$, and $Z_{6}^{2}=28.4$, respectively. The fact that the pulsed signal is less significant beyond $\sim 2.1$ $\mathrm{keV}$ can be explained by an increase of instrumental background along with a decrease of pulsar signal and, hence, a decreased signal-to-noise ratio compared to the $0.2-1.0 \mathrm{keV}$ and $1.0-2.1 \mathrm{keV}$ energy bands. An inspection of the pulse profiles for these two energy bands reveals that the emission from the narrow pulse peak appears only in the profile beyond $\sim 1$ $\mathrm{keV}$. Computing the fraction of pulsed photons, we find $32 \pm 4 \%$ for the total $0.2-10 \mathrm{keV}$ energy band. The pulsed fractions in the sub-bands $0.2-1.0 \mathrm{keV}, 1.0-2.1 \mathrm{keV}$, and $2.1-10$ $\mathrm{keV}$ are $24 \pm 5 \%, 44 \pm 6 \%$, and $17 \pm 17 \%$, respectively (errors represent the $1 \sigma$ confidence limits). For the narrow peak which appears at energies $\geq 1 \mathrm{keV}$, the pulsed fraction in the $1.0-2.1 \mathrm{keV}$ band is slightly higher than within the $0.2-1.0 \mathrm{keV}$ band, although the significance for this is at the $\sim 2 \sigma$ level only.

Computing the TOAs of the X-ray pulse we note that uncertainties of the XMM-Newton clock against UTCs are not relevant as those are on a scale of $\sim 100 \mu$ s (Becker et al. 2005 in prep.) and thus are a factor of $\sim 140$ smaller than the bin width of the X-ray pulse profile shown in Figure 7. The largest uncertainty from comparing radio with X-ray pulse arrival is the definition of a suitable reference point which we choose to be the center of mass of a 
pulse peak.

\subsection{SPECTRAL ANALYSIS}

\subsubsection{PSR B1929+10}

The energy spectrum of PSR B1929+10 was extracted from the MOS1/2 data by selecting all events detected in a circle of 40 arcsec radius, centered on the pulsar position. Using the XMM-Newton/EPIC-MOS model point spread function, $\sim 87 \%$ of all events of a point source are within this region. The background spectrum was extracted from a source free circular region of 30 arcsec radius, northwest from the pulsar at RA(2000) $19^{h} 32^{m} 08.59^{s}$, DEC $10^{\circ} 59^{\prime} 36.49^{\prime \prime}$. A second background spectrum was extracted from a circle of the same size but centered east from the pulsar at the position RA(2000) $19^{h} 32^{m} 19.5^{s}$, DEC $10^{\circ} 59^{\prime} 56.1^{\prime \prime}$. This second background spectrum was used to check the independence of the spectral results with respect to the selected background region.

For the EPIC-PN data we used an extraction radius of 30 arcsec centered on PSR B1929+10. This selection region includes $\sim 85 \%$ of the point source flux. Out-of-time events and a gradient of decreasing background towards the PN-CCD readout node requires that the background spectrum be selected from regions located at about the same CCD row level as the location of the pulsar. We therefore extracted two background spectra from source free circular regions of radius 30 arcsec about 1.5 arc-minute east and west of the pulsar position. The spectral results were found to be independent of the specific background region used.

Because of its unique soft response we made use of archival ROSAT PSPC data and extracted the pulsar spectrum from a 60 arcsec circular region in the PSPC. The background spectrum was extracted from an annulus of 70 arcsec and 100 arcsec inner and outer radius, respectively, centered on the pulsar.

In total, the extracted spectra include 2405 EPIC-PN source counts and 1536 EPICMOS1/2 source counts. The PN and MOS1/2 spectral data were dynamically binned so as to have at least 30 counts per bin. An additional 462 source counts recorded within $0.1-2.4$ $\mathrm{keV}$ by ROSAT were available for the joined ROSAT plus XMM-Newton spectral analysis of PSR B1929+10. As the energy resolution of the ROSAT PSPC was only $\sim 30 \%$ at $1 \mathrm{keV}$ (Briel et al. 1989) we binned the ROSAT data so as to have 4 independent spectral bins.

Model spectra were then simultaneously fitted to the ROSAT and XMM-Newton based pulsar spectra. An anomaly in the MOS2 spectral data below $\sim 0.8 \mathrm{keV}$ required exclusion 
of those spectral bins from the analysis.

Amongst the single component spectral models, a power law model was found to give the statistically best representation $\left(\chi^{2}=119.6\right.$ for 121 dof $)$ of the observed energy spectrum. A single blackbody model $\left(\chi^{2}=246.9\right.$ for 121 dof $)$ which was used by Yancopoulos et al. (1994) and Wang \& Halpern (1997) to describe the ROSAT and ASCA observed pulsar spectrum did not give acceptable fits and can finally be rejected. The best-fit power law spectrum and residuals are shown in Figure 10. Contour plots showing the relationship between the photon index and the column absorption for various confidence levels are shown in Figure 11.

The power law model yields a column absorption of $N_{H}=1.6_{-0.18}^{+0.2} \times 10^{21} \mathrm{~cm}^{-2}$, a photon-index $\alpha=2.72_{-0.09}^{+0.12}$ and a normalization of $8.1_{-0.5}^{+0.6} \times 10^{-5}$ photons $\mathrm{cm}^{-2} \mathrm{~s}^{-1} \mathrm{keV}^{-1}$ at $E=1 \mathrm{keV}$. The errors represent the $1 \sigma$ confidence range computed for one parameter of interest. These spectral parameters are in good agreement with the results obtained by Slowikowska et al. (2005) based on their joint analysis of ROSAT plus ASCA data. For the unabsorbed energy flux we measured $f_{x}=2.64_{-0.16}^{+0.12} \times 10^{-13} \mathrm{erg} \mathrm{s}^{-1} \mathrm{~cm}^{-2}$ in the $0.5-10$ $\mathrm{keV}$ band, yielding an X-ray luminosity of $L_{x}=4.11_{-0.16}^{+0.18} \times 10^{30} \mathrm{erg} \mathrm{s}^{-1}$. For the ROSAT energy band we measured the flux to be $f_{x}=8.59_{-1.36}^{+2.17} \times 10^{-13} \mathrm{erg} \mathrm{s}^{-1} \mathrm{~cm}^{-2}$, yielding an X-ray luminosity of $L_{x}=1.34_{-0.21}^{+0.34} \times 10^{31} \mathrm{erg} \mathrm{s}^{-1}$. These luminosities imply a rotational energy to X-ray energy conversion efficiency $L_{x} / \dot{E}$ of $1.1 \times 10^{-3}$ within $0.5-10 \mathrm{keV}$ and of $3.4 \times 10^{-3}$ within the ROSAT band.

In addition to the single component spectral models we tested two composite models consisting of either a blackbody plus power law component or of two blackbody components, respectively. The first model represents the scenario in which the X-ray emission of PSR B1929+10 originates from heated polar caps and from magnetospheric radiation processes. The double blackbody model implies that its X-ray emission would be entirely of thermal origin, e.g. with all the X-rays being emitted from heated polar caps with an anisotropic temperature distribution for which the model is approximated by two Planckian spectra with different emitting areas and temperatures.

Clearly, the statistical motivation to include an additional thermal component to the already excellent power law fit is very small. All combinations of blackbody normalizations and temperatures that were fitted gave reduced $\chi^{2}$-values which are not better than the fits to a power law model of the pulsar spectrum. The F-test statistic for adding the extra blackbody spectral component to the power law model, thus, is very low. Based upon the errors of the fitted spectral components all fits of this composite model yield only upper limits for a maximum thermal component which the power law model "accepts" before the fits become statistically unacceptable. The parameters obtained for the thermal component are thus 
intrinsically upper limits. The fitted parameters are a column absorption of $N_{H}=1.1_{-0.3}^{+0.6} \times$ $10^{21} \mathrm{~cm}^{-2}$, a photon-index $\alpha=2.45_{-0.21}^{+0.33}$ and a normalization of the power law component of $5.8_{-1.4}^{+1.7} \times 10^{-5}$ photons $\mathrm{cm}^{-2} \mathrm{~s}^{-1} \mathrm{keV}^{-1}$ at $E=1 \mathrm{keV}$. The blackbody temperature and the size of the projected emitting area are $k T \leq 0.28 \mathrm{keV}$ and $R_{b b} \leq 110 \mathrm{~m}$, assuming a pulsar distance of $361 \mathrm{pc}$.

For the unabsorbed energy flux and luminosity the model yields $f_{x}=2.37_{-0.56}^{+4.88} \times$ $10^{-13} \mathrm{erg} \mathrm{s}^{-1} \mathrm{~cm}^{-2}$ and $L_{x}=3.69_{-0.88}^{+7.6} \times 10^{30} \mathrm{erg} \mathrm{s}^{-1}$ in the $0.5-10 \mathrm{keV}$ band. For the $0.1-2.4 \mathrm{keV}$ energy band we compute the model flux to be $f_{x}=4.7_{-2}^{+9} \times 10^{-13} \mathrm{erg} \mathrm{s}^{-1} \mathrm{~cm}^{-2}$, yielding an X-ray luminosity of $L_{x}=7.38_{-3.1}^{+14.3} \times 10^{30} \mathrm{erg} \mathrm{s}^{-1}$.

In computing the relative contributions of the thermal and non-thermal spectral components and stretching the errors to the limits for the composite blackbody plus power law model, we find that no more than $\sim 40 \%$ of the detected X-ray flux could come from heated polar caps. For the best fit parameters only $\sim 7 \%$ could be emitted from these caps. However, the radius of $\leq 110 \mathrm{~m}$ which we computed for the maximum projected emitting area is not too different from the expected size of a polar cap. For comparison, defining the size of the presumed polar cap as the foot points of the neutron star's dipolar magnetic field, the radius of the polar cap area is given by $\rho=\sqrt{2 \pi R^{3} / c P}$ with $R$ being the neutron star radius, $\mathrm{c}$ the velocity of light and $\mathrm{P}$ the pulsar rotation period (see e.g. Michel 1991). For PSR B1929+10 with a rotation period of $226 \mathrm{~ms}$ this yields a polar cap radius of $\rho \sim 300 \mathrm{~m}$. A plot of the thermal and non-thermal spectral components and the combined model is shown in Figure 12.

With a spin-down age of $\sim 3 \times 10^{6}$ years PSR B1929+10 should still have some residual heat content from its birth event. Depending on the equation of state the surface temperature could still be in the range $\sim 1-3 \times 10^{5} \mathrm{~K}$ (cf. Becker \& Pavlov 2001 and references therein) and could contribute on a low level to the detected soft X-ray emission. To estimate the upper limit for the surface temperature of PSR B1929+10 we have fixed the blackbody normalization so that the emitting area corresponds to the surface of a $R=10 \mathrm{~km}$ neutron star and calculated the confidence ranges of the blackbody temperature by leaving all other parameters free. We find a $3 \sigma$ surface temperature upper limit of $T_{s}^{\infty}<4.5 \times 10^{5} \mathrm{~K}$ which is somewhat above the temperatures predicted by cooling models (e.g. Page \& Applegate 1992; Yakovlev et al. 1999) and, thus, may constrain only those thermal evolution models which predict extreme reheating.

The spectral model consisting of two thermal components describes the observed spectrum formally with comparable goodness of fit as the blackbody plus power law model. In comparison with a single blackbody fit the F-test statistic thus supports an addition of a second thermal component to the single blackbody model. However, inspection of the fit residu- 
als shows that the double blackbody model falls off rapidly beyond $\sim 5 \mathrm{keV}$ which causes the residuals beyond that energy to systematically lie above the zero line, albeit error bars are large. The fitted model parameters are a column absorption of $N_{H}=0.35_{-0.08}^{+0.11} \times 10^{21} \mathrm{~cm}^{-2}$, temperatures $k T_{1}=0.59_{-0.05}^{+0.06}, k T_{2}=0.20_{-0.2}^{+0.2}$, and projected emitting areas $R_{1}=9.8_{-1.8}^{+2.1} \mathrm{~m}$ and $R_{2}=92_{-11}^{+14} \mathrm{~m}$, respectively.

The spectral parameters of the various models fitted to the energy spectrum of PSR B1929+10 are summarized in Table 3 .

\subsubsection{Diffuse emission from the X-ray trail of PSR B1929+10}

The large collecting area of XMM-Newton allows, for the first time, a spectral analysis of the emission from the pulsar's X-ray trail. The satellite roll-angle and the EPIC-PN reduced field of view of $4.4 \times 4.4$ arcmin in SmallWindow mode caused only a small portion of the trail to be observed by the PN CCD \#4 in the short November 2003 observation. During the April 2004 observations the trail is outside the PNs field of view. Due to the increased instrument background towards the PN's readout node which is near to the location of the trail region in the CCD \#4 we did not include the PN data in the analysis of the diffuse emission. Both MOS cameras have covered a large portion of the trail in all observations (cf. Figure 3) so the analysis was restricted to the MOS1/2 data only.

The photon statistics of the diffuse trail emission are sufficient for a detailed spectral modeling only at regions near to the pulsar. We, therefore, extracted the X-ray spectrum from a circular region of 1 arcmin radius centered at $\mathrm{RA}(2000) 19^{h} 32^{m} 07.5^{s}$, DEC $10^{\circ} 58^{\prime} 32^{\prime \prime}$. Events from a second circular trail region of the same size but centered at $\operatorname{RA}(2000) 19^{h} 31^{m} 59.8^{s}$, DEC $10^{\circ} 57^{\prime} 02.3^{\prime \prime}$ were tried for the spectral analysis. However, the results were not meaningful because of a low signal-to-noise ratio which, for this region, is only $\sim 18 \%$. The background spectrum was extracted from a source free region located at $\operatorname{RA}(2000) 19^{h} 32^{m} 01.6^{s}$, DEC $10^{\circ} 59^{\prime} 36^{\prime \prime}$. About 800 source counts were available for the spectral analysis of the diffuse trail emission. We binned the spectrum dynamically so as to have at least 30 counts per bin.

The pulsar's X-ray trail is likely formed by a ram-pressure confined pulsar wind. Its Xray emission should arise from synchrotron radiation of relativistic electrons with a spectral shape characterized by a power law. To test this hypothesis we fitted a power law to the extracted trail spectrum and found the model describes the observed spectrum very well $\left(\chi^{2}=20.4\right.$ for 23 dof $)$. The best-fit power law spectrum and residuals are shown in Figure 13 . Contour plots showing the relationship between the photon index and the column absorption 
for various confidence levels are shown in Figure 14. The power law model yields a column absorption $N_{H}=0.6_{-0.6}^{+0.7} \times 10^{21} \mathrm{~cm}^{-2}$, a photon-index $\alpha=2.0_{-0.4}^{+0.4}$ and a normalization of $1.2_{-0.3}^{+0.5} \times 10^{-5}$ photons $\mathrm{cm}^{-2} \mathrm{~s}^{-1} \mathrm{keV}^{-1}$ at $E=1 \mathrm{keV}$. The energy flux and luminosities in the $0.5-10 \mathrm{keV}$ and $0.1-2.4 \mathrm{keV}$ bands are $f_{x}=5.3_{-2.2}^{+5.6} \times 10^{-14} \mathrm{erg} \mathrm{s}^{-1} \mathrm{~cm}^{-2}, L_{x}=$ $8.3_{-3.4}^{+8.7} \times 10^{29} \mathrm{erg} \mathrm{s}^{-1}$ and $f_{x}=5.8_{-2.3}^{+5.5} \times 10^{-14} \mathrm{erg} \mathrm{s}^{-1} \mathrm{~cm}^{-2}, L_{x}=9.1_{-3.6}^{+8.6} \times 10^{29} \mathrm{erg} \mathrm{s}^{-1}$, respectively. Accordingly, the 1 arcmin size portion of the diffuse nebula located near to the pulsar, but along its proper motion, direction radiates $2.1 \times 10^{-4} \dot{E}$ into the $0.5-10 \mathrm{keV}$ X-ray band and $2.3 \times 10^{-4} \dot{E}$ into the $0.1-2.4 \mathrm{keV}$ soft X-ray band.

For the second circular region which is located $\sim 2.5$ arcmin behind the pulsar along its proper motion direction we converted the background and vignetting corrected counting rate to an energy flux by assuming that the power law photon index is similar to the one we modeled from region one. It is not unlikely that this assumption is not justified. For example, Willingal et al. (2001) found in a detailed spectral analysis of the Crab nebula that its outer regions show the steepest spectrum. This indicates enhanced synchrotron losses of the electrons during their passage from the pulsar to the outskirts of the nebula. It is not unreasonable to assume that a similar behavior is valid for the electrons radiating in the trail of PSR B1929+10. The energy fluxes and luminosities we obtain for the second region are upper limits in this respect. For the $0.5-10 \mathrm{keV}$ and $0.1-2.4 \mathrm{keV}$ bands we find $f_{x} \sim 1.8 \times 10^{-14} \mathrm{erg} \mathrm{s}^{-1} \mathrm{~cm}^{-2}, f_{x} \sim 1 \times 10^{-14} \mathrm{erg} \mathrm{s}^{-1} \mathrm{~cm}^{-2}$ and $L_{x} \sim 2.8 \times 10^{29} \mathrm{erg} \mathrm{s}^{-1}$, $L_{x} \sim 1.6 \times 10^{29} \mathrm{erg} \mathrm{s}^{-1}$, respectively, which is $\sim 4-7 \times 10^{-5} \dot{E}$.

Wang, Li \& Begelman (1993) investigated the trail emission by assuming a synchrotron emission spectral shape for which they simulated a spectrum and compared model predictions vs. observed counting rates in these bands. Our findings based on a more detailed spectral modeling agree well with their results.

\subsection{MULTI-WAVELENGTH SPECTRUM}

In order to construct a broadband spectrum combining all spectral information available from PSR B1929+10 we adopted the radio spectrum from Maron et al. (2000) and plotted it in Figure 15 together with the XMM-Newton observed pulsar spectrum and the optical fluxes obtained from the Hubble Space Telescope observations by Pavlov et al. (1996) and Mignani et al. (2002).

The flux density in the radio part of the spectrum, which should be due to coherent radiation, is several orders of magnitude greater than the extrapolated optical or X-ray flux densities. Extrapolating the power law spectrum which describes the XMM-Newton data to 
the optical bands yields a photon flux which exceeds the flux measured in the near-UV bands (Pavlov et al. 1996; Mignani et al. 2002) by more than an order of magnitude. This suggests that the broadband spectrum, if entirely non-thermal, has to break somewhere before or in the soft channels of the X-ray spectrum. To test this hypothesis we have fitted a broken power law model to the XMM-Newton and optical data and found it provides an excellent description $\left(\chi^{2}=121.16\right.$ for 120 dof $)$ of both spectral data sets. A broken power law model which is in agreement with the optical observations and the XMM-Newton data has its break point fitted at $E_{\text {break }}=0.83_{-0.03}^{+0.05} \mathrm{keV}$. The photon-index for $E<E_{\text {break }}$ and $E>E_{\text {break }}$ is found to be $\alpha_{1}=1.12_{-0.03}^{+0.02}$ and $\alpha_{2}=2.48_{-0.07}^{+0.08}$, respectively, with a normalization of $8.2_{-0.7}^{+1.0} \times 10^{-5}$ photons $\mathrm{cm}^{-2} \mathrm{~s}^{-1} \mathrm{keV}^{-1}$ at $1 \mathrm{keV}$. The model predicted column absorption is $N_{H}=0.52_{-0.06}^{+0.12} \times 10^{21} \mathrm{~cm}^{-2}$ which is smaller than the single power law model prediction, but is still compatible with our fit to the diffuse trail emission.

\section{SUMMARY \& DISCUSSION}

We have investigated the emission properties of PSR B1929+10 and its X-ray trail in a multi-wavelength study using XMM-Newton, the ESO New Technology Telescope (NTT) in La Silla (Chile), the Hubble Space Telescope, the Effelsberg 100-m Radio Telescope and the Jodrell Bank Radio Observatory.

In X-rays, PSR B1929+10 is the brightest pulsar among all members of its class. For about 10 years it, therefore, was the only X-ray detected old rotation-powered pulsar for which some details on its emission properties were known - though this picture has been revised thoroughly in our multi-wavelength study of the old rotation-powered pulsars (cf. Becker et al. 2004; 2005).

Clearly, the study of old rotation-powered pulsars is in the domain of XMM-Newton which was designed and built to study faint sources in the X-ray sky. Its sensitivity allowed, for the first time, a more detailed study of this interesting group of pulsars. As for PSR B0628-28 (Becker et al. 2005; Tepedelenlioglu \& Ögelman 2005), PSR B0950+08, PSR B0823+26 and PSR J2043+2740 (Becker et al 2004; Pavlov \& Zavlin 2004) which all were observed by XMM-Newton in recent observations we found the temporal and spectral X-ray emission properties of PSR B1929+10 in excellent agreement with a non-thermal and, thus magnetospheric radiation dominated emission scenario. Its X-ray spectrum is best described by a single power law model with a photon index of $2.72_{-0.09}^{+0.12}$. The flux contribution from thermal emission of heated polar caps of $\sim 7 \%$ is inferred from the best fit composite Planckian and power law spectral model. However, a pure thermal emission spectrum consisting of two Planckian spectra is regarded as unlikely. Taking the optical spectral data into account 
a broken power law with $E_{\text {break }}=0.83_{-0.03}^{+0.05} \mathrm{keV}$ and the photon-index $\alpha_{1}=1.12_{-0.03}^{+0.02}$ and $\alpha_{2}=2.48_{-0.07}^{+0.08}$ is able to describe the emission in both spectral ranges entirely in terms of a non-thermal magnetospheric origin.

The X-ray pulse profile observed in the $0.2-10 \mathrm{keV}$ band is found to be markedly different from the broad sinusoidal pulse profile seen in the low statistic ROSAT data. Fitting Gaussians to the X-ray light curve indicates the possible existence of three pulse components. A small narrow pulse, characterized by energies greater than $1 \mathrm{keV}$, is found to lead the radio main pulse by $\sim 20^{\circ}$. Two larger pulses, observed in all three energy bands, follow this small pulse. These three pulses are roughly separated by about the same phase cycle (cf. Figure $5)$. The fraction of pulsed photons in the $0.2-10 \mathrm{keV}$ band is $32 \pm 4 \%$. For the sub-bands $0.2-1.0 \mathrm{keV}$ and $1.0-2.1 \mathrm{keV}$ the pulsed fraction is $24 \pm 5 \%$ and $44 \pm 6 \%$, respectively, indicating a mild energy dependence on a $\sim 2 \sigma$ level.

The existence of diffuse emission with a trail morphology lying in a direction opposite to the motion of the pulsar is confirmed in our XMM-Newton observation without any doubt and provides a unique opportunity to probe the pulsar and its environment. The discovery of its radio counterpart with the Effelsberg Radio Telescope at $11 \mathrm{~cm}$ wavelength is exciting and provides an important and independent evidence for its existence. No diffuse emission from the pulsar trail is detected in $\mathrm{H}_{\alpha}$. In the XMM-Newton MOS1/2 images the trail is seen down to the edge of the detector, i.e. its length is approximately 15 arcmin. The trail length seen in the $11 \mathrm{~cm}$ radio map is of comparable size and structure as its X-ray counterpart. Since its X-ray emission has a hard spectrum characterized by a power law photon index of $\sim 2$, the emission is non-thermal and is likely to be produced from the synchrotron process of highly relativistic electrons in the shocked region between the pulsar wind and surrounding interstellar medium. A physical description of PSR B1929+10's X-ray trail based on the ROSAT findings has been discussed by Wang, Li, \& Begelman (1993) in terms of an outflow collimated within the pulsar's cavity created by its motion.

The properties of the distorted wind nebula can, alternatively, be inferred under the assumption that the electron lifetime due to synchrotron losses, $\tau_{\text {syn }}$, is comparable to the timescale for the passage of the pulsar over the length of its X-ray trail. Such a hypothesis has also been considered, for consistency, by Caraveo et al. (2003) in their model analysis of a similar X-ray trail observed in the Geminga pulsar. For PSR 1929+10, the angular extent of the trail is not definitively determined, but it is likely greater than 15 arcmin. For an assumed distance of $361 \mathrm{pc}$, the linear scale, $d$, corresponding to this angular scale is $1.6 \mathrm{pc}$. An estimate of the flow time, $t_{\text {flow }}$, can be obtained, for the proper motion measured by Chatterjee et al. (2004) and resulting velocity of $v_{p}$ of $177 \mathrm{~km} \mathrm{~s}^{-1}$, leading to $t_{\text {flow }} \sim 8600 \mathrm{yrs}$, This is about a factor of 9 times longer than the comparable timescale 
found for the Geminga pulsar (see Caraveo et al. 2003). To determine the consistency of our interpretation, the magnetic field in the shocked region can be estimated by equating $t_{s y n}$ to $t_{\text {flow }}$. Here $\tau_{\text {syn }}=6 \pi m_{e} c / \gamma \sigma_{T} B^{2} \sim 10^{5} B_{\mu G}^{-3 / 2}\left(h \nu_{X} / \mathrm{keV}\right)^{-1 / 2}$ yr where $\gamma$ is the Lorentz factor of the wind, taken to be equal to $10^{6}, \sigma_{T}$ is the Thompson cross section, and $B_{\mu G}$ is the magnetic field in the emission region in micro gauss. The inferred magnetic field strength in the emitting region is $\sim 5 \mu \mathrm{G}$. Given the magnetic field strength estimates in the interstellar medium ( $\sim 2-6 \mu \mathrm{G}$; see Beck et al. 2003), and the expected compression of the field in the termination shock by about a factor of 3 (Kennel \& Coroniti 1984), our estimates for the magnetic field in the emitting region of the pulsar wind nebula are in approximate accord.

The X-ray luminosity and the spectrum of the emitted radiation can be estimated using a simple, one-zone model for the emission nebula powered by the pulsar wind as developed by Chevalier (2000). To determine the characteristic properties of the emission a comparison of the cooling frequency, $\nu_{c}$, for which the electrons can radiate their energy in the pulsar trail, with the observing frequency, $\nu_{X}$ is necessary. The cooling frequency can be expressed as $\nu_{c}=\frac{e}{2 \pi m_{e} c B^{3}}\left(\frac{6 \pi m_{e} c}{\sigma_{T} \tau_{s y n}}\right)^{2}$. Substitution of the inferred magnetic strength and the electron cooling timescale due to synchrotron radiation yields $\nu_{c}=1.8 \times 10^{17} \mathrm{~Hz}$. Since $\nu_{X}>\nu_{c}$, the electrons are able to radiate their energy in the pulsar trail and the photon index, $\Gamma$, is related to the power law index, $p$, of the electron energy distribution, $N(\gamma) \propto \gamma^{-p}$, in the form $\Gamma=(p+2) / 2$. Based on the theoretical work on highly relativistic shocks (Bednarz \& Ostrowski 1998, Lemoine \& Pelletier 2003), we adopt $p=2.2$, yielding $\Gamma=2.1$. To be consistent with our interpretation of emission taking place in the fast cooling region, the observed value of $\Gamma$ should exceed 2 , which is consistent with the observed value of $\Gamma=2_{-0.4}^{+0.4}$.

The luminosity of the radiating electrons in the nebula can be calculated from the luminosity per unit frequency given by

$$
\begin{gathered}
L_{\nu}=\frac{1}{2}\left(\frac{p-2}{p-1}\right)^{p-1}\left(\frac{6 e^{2}}{4 \pi^{2} m_{e} c^{3}}\right)^{(p-2) / 4} \epsilon_{e}^{p-1} \epsilon_{B}^{(p-2) / 4} \gamma_{w}^{p-2} \\
R_{s}^{-(p-2) / 2} \dot{E}^{(p+2) / 4} \nu^{-p / 2} .
\end{gathered}
$$

where $R_{s}$ is the distance of the shock from the pulsar expressed as $R_{s}=\left(\dot{E} / 2 \pi \rho v_{p}^{2} c\right)^{1 / 2} \sim$ $10^{16} \dot{E}_{33}^{1 / 2} n^{-1 / 2} v_{p, 100}^{-1} \mathrm{~cm}$. Here $v_{p, 100}$ is the velocity of the pulsar in units of $100 \mathrm{~km} \mathrm{~s}^{-1}, \dot{E}_{33}$ is the spin down power of the pulsar in units of $10^{33} \mathrm{ergs} \mathrm{s}^{-1}$, and $n$ is the number density of the interstellar medium in units of $1 \mathrm{~cm}^{-3}$. The spin down power of $3.89 \times 10^{33} \mathrm{ergs} \mathrm{s}^{-1}$ and a density of $1 \mathrm{~cm}^{-3}$ yields a shock radius of $R_{s} \sim 1.1 \times 10^{16} \mathrm{~cm}$. Assuming energy equipartition between the electron and proton fractional energy densities so that $\epsilon_{e} \sim 0.5$, and a fractional energy density of the magnetic field $\epsilon_{B} \sim 0.01$ (see Cheng, Taam, \& Wang 2004), the corresponding luminosity given as $\nu L_{\nu}$ is $\sim 1.5 \times 10^{30} \mathrm{ergs} \mathrm{s}^{-1}$ or $\sim 3 \times 10^{-4} \dot{E}$. 
In view of the observational uncertainties, this is consistent with the observed values of $8.3_{-3.4}^{+8.7} \times 10^{29} \operatorname{ergs~s}^{-1}(0.5-10 \mathrm{keV})$ and $9.1_{-3.6}^{+8.6} \times 10^{29} \operatorname{ergs~s}^{-1}(0.1-2.4 \mathrm{keV})$.

Pulse profiles very similar in shape to that found in PSR B1929+10 are observed from the old pulsars PSR B0950 - 08 and PSR B0628 - 28 (Becker et al. 2004; 2005). The spectra of all these X-ray pulsars are dominated by non-thermal magnetospheric radiation processes, likely with some thermal contribution from heated polar caps at the few percent level. Various theoretical models have been developed to explain the observed high energy emission observed in younger pulsars like the Crab and Vela-pulsar which all appear to be seen not only in X-rays but also in the gamma-ray band. Can the generic features of these models explain the emission properties we observe from the older pulsars as well?

It is commonly believed that the non-thermal X-ray photons are emitted by relativistic charged particles in the pulsar magnetosphere. These relativistic particles could be accelerated in a polar gap region (e.g. Harding 1981; Zhang \& Harding 2000) or in the outer gap region (e.g. Cheng, Ho \& Ruderman 1986). The light curves of high energy radiation have been simulated using three dimensional models (e.g. Chiang \& Romani 1992; Yadigaroglu \& Romani 1995; Cheng, Ruderman \& Zhang 2000; Dyks \& Rudak 2004; Dyks, Harding \& Rudak 2004). In general, light curves with a double-pulse structure are the most common feature of both polar gap and outer gap models. Although the detailed theoretical description of the light curves depends sensitively on the model, the suggestive triple-peak feature in the X-ray light curve of PSR B1929+10 and their relative phase separation may provide diagnostic information about the location of the emission regions. For example, since the plane of the magnetic axis and the rotation axis should lie in the middle of the two radio pulses, it will lie roughly in the middle of the first small peak and second large X-ray pulse. A triple-peak light curve feature can be observed if the inclination angle of the magnetic axis with respect to the rotation axis is sufficiently large. For inclination angles larger than $70^{\circ}$ (e.g. Dyks, Harding \& Rudak 2004), radiation from both poles of a pulsar could be observed. In the case of PSR B1929+10, the inclination angle is only $\sim 36^{\circ}$, and it is very unlikely that both poles can be directly observed. To be consistent with this small inclination angle, it is possible that one of the three peaks is emitted from a hot polar cap. In Figure 9, it can be seen that the proposed second and third peak appear in both the $0.2-1.0 \mathrm{keV}$ and 1.0 - $2.1 \mathrm{keV}$ energy bands with very similar phase separation whereas the first small narrow peak only appears beyond $\sim 1 \mathrm{keV}$. We note that these features are only present in the XMM-Newton data since the low count rate in previous ROSAT and ASCA observations makes the first narrow peak insignificant whereas the proposed double-peak or possibly single broad pulse feature remains in the energy range of $0.5-5.0 \mathrm{keV}$ (Yancopoulos et al. 1994; Slowikowska et al. 2004). It is therefore possible that the first narrow peak may arise from a different region. That it originates from a heated polar cap of $\mathrm{kT} \sim 1 \mathrm{keV}$, however, is very 
unlikely by its narrowness as strong gravitational light bending would smear out the thermal pulse from the surface (Page 1995; Tang \& Cheng 2001).

In the following we seek an alternative interpretation and calculate a theoretical Xray light curve in the framework of the outer-gap model. Here, the pulsar magnetosphere is approximated as a rotating magnetic dipole, where the spin axis of the pulsar is on the z-axis and the magnetic axis is in the (x.z) plane. High energy photons are assumed to be emitted along the magnetic field lines, and aberration and travel time effects are taken into account for the photon emission. The details of the three dimensional magnetospheric structure can be found in Cheng, Ruderman \& Zhang (2000). Everett \& Weisberg (2001) have reported from radio polarization data that the inclination and impact angles of PSR B1929+10 are $36^{\circ}$ and $26^{\circ}$ respectively. For such small inclination and impact angles, at most two pulses with a phase separation of $\sim 180^{\circ}$ can be produced if there is only outgoing current. In this case, one pulse arises from a region near one polar cap and another pulse arises from a region near the light cylinder, but associated with another magnetic pole. Here, relativistic charges moving along the curved field can radiate high energy curvature photons, which can be converted into electron/positron pairs via either magnetic pair production or photon-photon pair creation when colliding with the soft X-rays from the neutron star surface. It is not unreasonable that an incoming current can exist, at least, within a few hundred stellar radii. Since the radiation is expected to be emitted from open field lines, the coordinate values $\left(x_{0}, y_{0}, z_{0}\right)$ of the last closed field lines at the stellar surface must be determined. The coordinate values $\left(x_{0}^{\prime}, y_{0}^{\prime}, z_{0}^{\prime}\right)$, where $x_{0}^{\prime}=a_{1} x_{0}, y_{0}^{\prime}=a_{1} y_{0}$ and $z_{0}^{\prime}=\left[1-\left(x_{0}^{, 2}+y_{0}^{\prime 2}\right)\right]^{1 / 2}$, then represent an open field line surface for a given value of $a_{1}$. For simplicity, we choose $a_{1}=0.97$, which is very close to the first open field lines $\left(a_{1}=1.0\right)$. In Figure 16, we find that two large pulses and one small pulse are produced. The first large pulse is a result of the incoming current toward the south pole and another pulse is produced by the outgoing current from the north pole. The small pulse is produced by outgoing current near the light cylinder. We note that the phase separation of these three pulses roughly corresponds to the phase separation of the three pulses in the observed X-ray light curve. Here, the X.Z plane is choosen to lie at the zero-phase position in Figure 16. On the other hand, in Figure 7, the x.z plane should lie in the middle between the two radio pulses. Therefore, the simulated X-ray light curves roughly reproduce the phase relative to the radio pulses within a phase error of about \pm 0.1 which is well within the observed uncertainties.

Although we can roughly reproduce the X-ray light curves, more detailed theoretical modeling must be carried out before we can confirm that the X-ray emission regions correspond to the regions proposed above. In Figure 9, it can be seen that the X-ray light curves are energy dependent. Our simple light curve simulation is insufficiently detailed to provide an explanation for the appearance of the first narrow peak only at energies greater 
than $\sim 1 \mathrm{keV}$. For example, this peak arises from field lines very near the light cylinder in our simulations. X-rays are likely emitted by secondary electron/positron pairs, however, the production of electron/positron pairs in this region is quite difficult. Furthermore, relativistic charged particles created from regions near the star and moving along field lines will primarily radiate their energies at gamma-ray energies through the curvature radiation process instead of radiating in the X-ray regime.

These theoretical issues will necessitate future investigations which hopefully will be complemented by X-ray light curves and spectra having even better photon statistics than the ones which we have obtained in this first XMM-Newton observations of PSR B1929+10. The results we obtained from the X-ray trail along with the discovery of its radio counterpart are exciting. Follow up radio observations of the trail at different wavelengths are currently set up in order to obtain the spectral index in the radio regime which will provide additional constraints on its nature.

\section{REFERENCES}

Beck, R., Shukurov, A., Sokoloff, D., \& Wielebinksi, R. 2003. A\&A, 411, 99

Becker, W., Jessner, A., Kramer, M., Testa, V., Howaldt C., 2005, ApJ, in press (astro$\mathrm{ph} / 0505488)$

Becker, W., Weisskopf, M.C., Tennant, A.F., Jessner, A., Dyks, J., Harding, A.K., Zhang, S.N., 2004, ApJ, 615, 908

Becker, W., Aschenbach, B., 2002, in Proceedings of the WE-Heraeus Seminar on Neutron Stars, Pulsars and Supernova remnants, Eds. W.Becker, H.Lesch \& J.Trümper, MPEReport 278, 64, (available from astro-ph/0208466)

Becker, W., Pavlov, G.G., 2001, in The Century of Space Science, Eds. J.Bleeker, J.Geiss \& M.Huber, Kluwer Academic Publishers, p721 (available from astro-ph/0208356).

Becker, W. \& Trümper, J. 1999, A\&A, 341, 803

Becker, W., Trümper, J., 1997, A\&A, 326, 682

Bednarz, J. \& Ostrowski, M. 1998, Phys. Rev. Lett., 80, 3911

Briel, U.G., Burkert, W., Pfeffermann, E., 1989, in EUV, X-ray, and Gamma-ray Instrumentation for Astronomy and Atomic Physics, eds C.J. Hailey \& O.H.W. Siegmund, SPIE Vol. 1159, 263 
Buccheri R., De Jager O.C., in Timing Neutron Stars, Eds. H.Ögelman, E.P.J. van den Heuvel, p95, Kluwer Academic Publishers, 1989

Caraveo, P. A., Bignami, G. F., DeLuca, A., Mereghetti, S., Pellizzoni, A., Mignani, R., Tur, A., \& Becker, W. 2003, Sci., 301, 1345

Chatterjee, S., Cordes, J.M., Vlemmings, W.H.T., Arzoumanian, Z., Goss, W.M., Lazio, T.J.W., ApJ, 2004, 604, 339

Cheng, K. S., Ho, C., Ruderman, M. 1986, ApJ, 300, 500

Cheng, K. S., Ruderman, M. A., Zhang, L. 2000, ApJ, 537, 964

Cheng, K. S., Taam, R. E., Wang, W., 2004, ApJ, 617, 480

Chevalier, R. A., 2000, ApJ, 539, L45

Chiang, J., \& Romani, R. W., 1992, ApJ, 400,629

Cordes, J.M., Lazio, T.J.W., 2002, astro-ph/0207156

De Jager O.C., 1987, thesis, Potchefstromm University for Christian Higher Education, South Africa

De Luca, A., Caraveo, P.A., Mereghetti, S., Negroni, M., Bignami, G.F., 2005, ApJ, 623, 1051

Downs, G.S., Reichley, P.E., 1983, ApJS, 53,169

Dyks, J., Harding, A. K., \& Rudak, B. 2004, ApJ, 606, 1125

Dyks, J., \& Rudak, B. 2004, AdSpR, 33, 581

Harding, A.K., Muslimov, A.G., 2003, in the proceedings of Pulsars, AXPs and SGRs Observed by BeppoSAX and Other Observatories, astro-ph/0304121

Harding, A.K., Muslimov, A.G.,2002, ApJ, 568, 862

Harding, A.K., Muslimov, A.G.,2001, ApJ, 556, 1001

Harding, A. K. 1981, ApJ, 245, 267

Helfand, D.J., 1983, in Supernova Remnants and Their X-ray Emission, Eds. J.Danziger and P.Gorenstein, Proceedings of the IAU Symposium No. 101, p471 
Hobbs, G., Lyne, A. G., Kramer, M., Martin, C. E., Jordan, C. A., 2004, MNRAS, 353, 1311

Kennel, C.F., Coroniti, F.V. 1984, ApJ, 283, 694

Kirsch, M.G.F., Becker, W., Benlloch-Garcia, S., et al., in X-Ray and Gamma-Ray Instrumentation for Astronomy XIII, eds Flanagan, K.A. \& Siegmund, O.H.W., SPIE, Volume 5165, 85, 2004

Kramer, M., Jessner, A., Doroshenko, O., Wielebinski, R., 1997, A\&A, 488, 364

Kramer, M., Wielebinski, R., Jessner, A., Gil, J.A., Seiradakis, J.H., 1994, Astr. \& Astrophys. Suppl., 107, 515

Krautter, J., Zickgraf, F.-J., Appenzeller, I., Thiering, I., Voges, W., Chavarria, C., Kneer, R., Mujica, R., Pakull, M.W., Serrano, A., Ziegler, B., 1999, A\&A, 350, 743

Kouwenhoven, M.L.A., van den Berg, M.C., 2001, A \& A, 367, 931

Lemoine, M., Pelletier, G. 2003, ApJ, 589, L73

Manning, R., Willmore, P., 1994, MNRAS, 266, 635

Maron et al., Astron.Astrophys. Suppl. Ser. 147, 195, 2000

Michel, F.C., 1991, Theory of Neutron Star Magnetospheres, University of Chicago Press, Chicago, IL

Mignani, R.P., De Luca, A., Caraveo, P.A., Becker, W., ApJ, 2002, ApJ, 580L, 147

Page, D. 1995, ApJ, 442, 273

Page, D., Applegate, J.L. 1992, ApJ, 394, L17

Pavlov, G.G., Stringfellow, G.S., Cordova, F.A., 1996, ApJ, 467,370

Reich, W., Fuerst, E., Reich, P., Reif, K., 1990, A\&A, Suppl. 85, 633

Saito, Y., 1998, PhD Thesis, ISAS Research Note 643

Seward, F.D., Wang, Z.R., 1988, ApJ, 332, 199

Schlegel, D., Finkbeiner, D., Davis, M., ApJ, 1998, 500, 525.

Slowikowska, A., Kuiper, L., Hermsen, W., 2005, A\&A, 434, 1097

Standish, E.M., 1982 A\&A, 114,297 
Sun, X., Trümper, J., Dennerl, K., Becker, W., 1993, IAU circ. 5895

Tang, A. P. S., \& Cheng, K. S. 2001, ApJ, 549, 1039

Tepedelenlioglu, E., Ögelman, H.B., 2005, ApJ, submitted (astro-ph/0505461)

Wang, D., Halpern, J.P., 1997, ApJ, 482, L159

Wang, Q. D., Li, Z. Y., \& Begelman M. C. 1993, Nature, 364, 127

Willingale, R., Aschenbach, B., Griffiths, R. G., Sembay, S., Warwick, R. S., Becker, W., Abbey, A. F., Bonnet-Bidaud, J.-M., 2001, A\&A, 365, L212

Yadigaroglu, I.-A., \& Romani, R. W. 1995, ApJ, 449, 211

Yakovlev, D.G., Levenfish, K.P., Shibanov, Yu.A. 1999, Physics-Uspekhi, 169, 825

Yancopoulos, S., Hamilton, T.T., Helfand, D.J., 1994, ApJ, 429, 832

Zavlin, V.E., Pavlov, G.G., 2004, ApJ, 616, 452

Zickgraf, F.-J., Krautter, J., Reffert, S., Alcala, J.M., Mujica, R., Covino, E., Sterzik, M.F., 2005, A\&A, 433, 151

Zhang, B., Harding, A.K., 2000, ApJ, 532, 1150

WB would like to thank Hermann Brunner for his help in the X-ray image reconstruction. We are grateful to Olaf Maron for the use of his database of pulsar radio fluxes and E. Fürst for help with the $11 \mathrm{~cm}$ Effelsberg radio survey. KSC and JJJ are partially supported by a RGC grant of Hong Kong Government and RET acknowledges support from the NSF through grant AST-0200876. Optical observations were made with ESO Telescopes at the La Silla Observatories under program 077.D-0794(A). 
Table 1. Ephemerides of PSR B1929+10

\begin{tabular}{|c|c|}
\hline Right Ascension (J2000) & $19^{h} 32^{m} 13^{s} .983 \pm 00^{s} .002$ \\
\hline 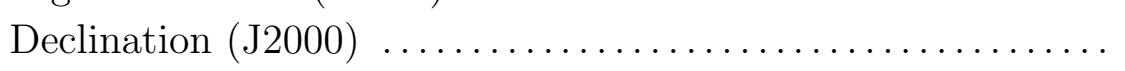 & $+10^{d} 59^{m} 32^{s} .41 \pm 00^{s} .07$ \\
\hline First date for valid parameters (MJD) & 52929 \\
\hline Last date for valid parameters (MJD) & 53159 \\
\hline Infinite-frequency geocentric pulse arrival time ${ }^{a}$ (MJD, UTC) & 53120.000002393 \\
\hline Pulsar rotation period $(s)$ & 0.2265182954 \\
\hline Pulsar rotation frequency $\left(s^{-1}\right)$ & 4.4146544466756 \\
\hline First derivative of pulsar frequency $\left(s^{-2}\right)$ & $-2.26998 \times 10^{-14}$ \\
\hline Second derivative of pulsar frequency $\left(s^{-3}\right)$ & $2.72 \times 10^{-26}$ \\
\hline Spin-down age $\left(y r / 10^{6}\right)$ & 3.09 \\
\hline Spin-down energy $\left(\mathrm{erg} / \mathrm{s} / 10^{33}\right)$ & 3.89 \\
\hline Inferred Magnetic Field $\left(G / 10^{12}\right)$ & 0.5129 \\
\hline Dispersion Measure $\left(\mathrm{pc} / \mathrm{cm}^{3}\right) \ldots$ & 3.178 \\
\hline Distance $^{b}(\mathrm{pc}) \ldots \ldots \ldots \ldots \ldots$ & $361_{-8}^{+10}$ \\
\hline
\end{tabular}

Note. -

${ }^{a}$ The integer part of this time is the barycentric (TDB) epoch of RA, DEC, f, $\dot{f}, \ddot{f}$.

${ }^{b}$ Distance based on radio parallax according to Chatterjee et al (2004). 
Table 2. Instrument setups, filter usage, start time, durations, and effective exposures of the XMM-Newton observations of PSR B1929+10.

\begin{tabular}{|c|c|c|c|c|c|}
\hline Detector & Mode & Filter & $\begin{array}{c}\text { Start time } \\
(\mathrm{UTC})\end{array}$ & $\begin{array}{c}\text { Duration } \\
\text { sec }\end{array}$ & $\begin{array}{c}\text { eff. Exp. } \\
\text { sec } \\
\end{array}$ \\
\hline \multicolumn{6}{|c|}{ SEQ: 0718_0113051301 } \\
\hline EMOS1 & PrimeFullWindow & Medium & 2003-11-10T02:11:28 & 10677 & 7345.1 \\
\hline EMOS2 & PrimeFullWindow & Medium & 2003-11-10T02:11:24 & 10668 & 7893.3 \\
\hline EPN & PrimeSmallWindow & Medium & 2003-11-10T02:16:44 & 10470 & 6994.0 \\
\hline \multicolumn{6}{|c|}{ SEQ: 0718_0113051401 } \\
\hline EMOS1 & PrimeFullWindow & Medium & 2004-04-27T13:13:15 & 21665 & 13499.2 \\
\hline EMOS2 & PrimeFullWindow & Medium & 2004-04-27T13:13:09 & 21676 & 14351.9 \\
\hline EPN & PrimeSmallWindow & Medium & 2004-04-27T13:18:29 & 21470 & 10556.0 \\
\hline \multicolumn{6}{|c|}{ SEQ: 0718_0113051501 } \\
\hline EMOS1 & PrimeFullWindow & Medium & 2004-04-29T13:13:44 & 22673 & 10271.6 \\
\hline EMOS2 & PrimeFullWindow & Medium & 2004-04-29T13:13:44 & 22678 & 6353.6 \\
\hline $\mathrm{EPN}$ & PrimeSmallWindow & Medium & 2004-04-29T13:19:06 & 22471 & 6268.3 \\
\hline
\end{tabular}


Table 3. Models and parameters as fitted to the energy spectrum of PSR B1929+10 and its X-ray trail.

\begin{tabular}{|c|c|c|c|c|c|c|}
\hline model $^{a}$ & $\chi_{\nu}^{2}$ & $\nu$ & $\begin{array}{c}N_{H} / 10^{21} \\
\mathrm{~cm}^{-2} \\
\end{array}$ & $k T^{b} / \alpha$ & $\begin{array}{c}\text { Normalization at } 1 \mathrm{keV} \\
\text { Photons } / \mathrm{keV} / \mathrm{cm}^{2} / \mathrm{s}\end{array}$ & $\begin{array}{c}\text { Radius }^{c} \\
\mathrm{~m}\end{array}$ \\
\hline PL & 0.989 & 121 & $1.6_{-0.18}^{+0.2}$ & $2.72_{-0.09}^{+0.12}$ & $8.1_{-0.5}^{+0.6} \times 10^{-5}$ & \\
\hline $\mathrm{BB}+\mathrm{PL}$ & 0.997 & 119 & $1.1_{-0.3}^{+0.6}$ & $\leq 0.28 / 2.45_{-0.21}^{+0.33}$ & $5.8_{-1.4}^{+1.7} \times 10^{-5}$ & $\leq 106$ \\
\hline $\mathrm{BB}+\mathrm{PL}$ & 0.996 & 120 & $1.6_{-0.2}^{+0.3}$ & $\leq 0.04 / 2.73_{-0.1}^{+0.13}$ & $8.2_{-0.6}^{+0.8} \times 10^{-5}$ & 10000 \\
\hline $\mathrm{BKNPL}^{d}$ & 1.010 & 120 & $0.52_{-0.06}^{+0.12}$ & $\begin{array}{l}\alpha_{1}=1.12_{-0.03}^{+0.02} \\
\alpha_{2}=2.48_{-0.07}^{+0.08}\end{array}$ & $8.2_{-0.7}^{+1.0} \times 10^{-5}$ & \\
\hline $\mathrm{BB}+\mathrm{BB}$ & 1.069 & 119 & $0.35_{-0.08}^{+0.11}$ & $\begin{array}{l}\mathrm{kT}_{1}=0.59_{-0.05}^{+0.06} \\
\mathrm{kT}_{2}=0.20_{-0.2}^{+0.2}\end{array}$ & & $\begin{array}{l}9.8_{-1.8}^{+2.1} \\
92_{-11}^{+14}\end{array}$ \\
\hline
\end{tabular}

Diffuse emission from the pulsar's X-ray trail
PL
$0.8853 \quad 23$
$0.6_{-0.6}^{+0.7}$
$2.0_{-0.4}^{+0.4}$
$1.2_{-0.3}^{+0.5} \times 10^{-5}$

Note. -

${ }^{a} \mathrm{BB}=$ blackbody, $\mathrm{PL}=$ power law, $\mathrm{BKNPL}=$ broken power law

${ }^{b}$ The entry in this column depends on the spectral model - it is the temperature $k T$ in $\mathrm{keV}$ or the photon index $\alpha$.

${ }^{c}$ For thermal models for which we computed or fixed the radius of the emitting area we assumed a pulsar distance of $361 \mathrm{pc}$.

$d$ The broken power law model has been fitted to the combined X-ray and optical spectral data. The break energy is fitted to be $E_{\text {break }}=0.83_{-0.03}^{+0.05} \mathrm{keV}$ 


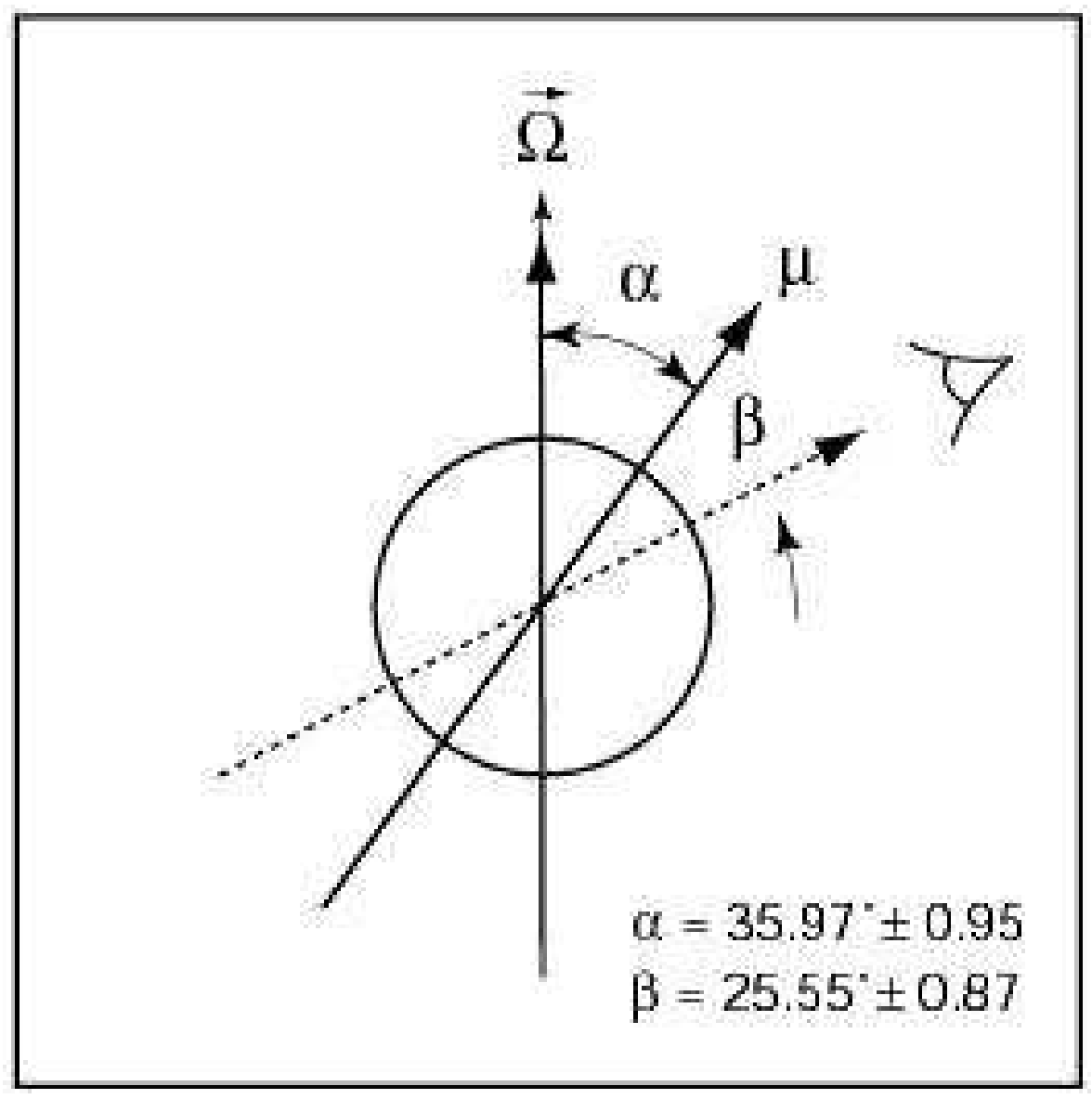

Fig. 1. - Emission beam geometry of PSR B1929+10 as deduced from fitting the rotating vector model to the polarization angle swing observed at radio frequencies (e.g. Everett \& Weisberg 2001). $\alpha$ is the inclination of the magnetic axis, $\beta$ the minimum angle between the magnetic axis and the line of sight. $\vec{\Omega}$ is the rotation axis. 


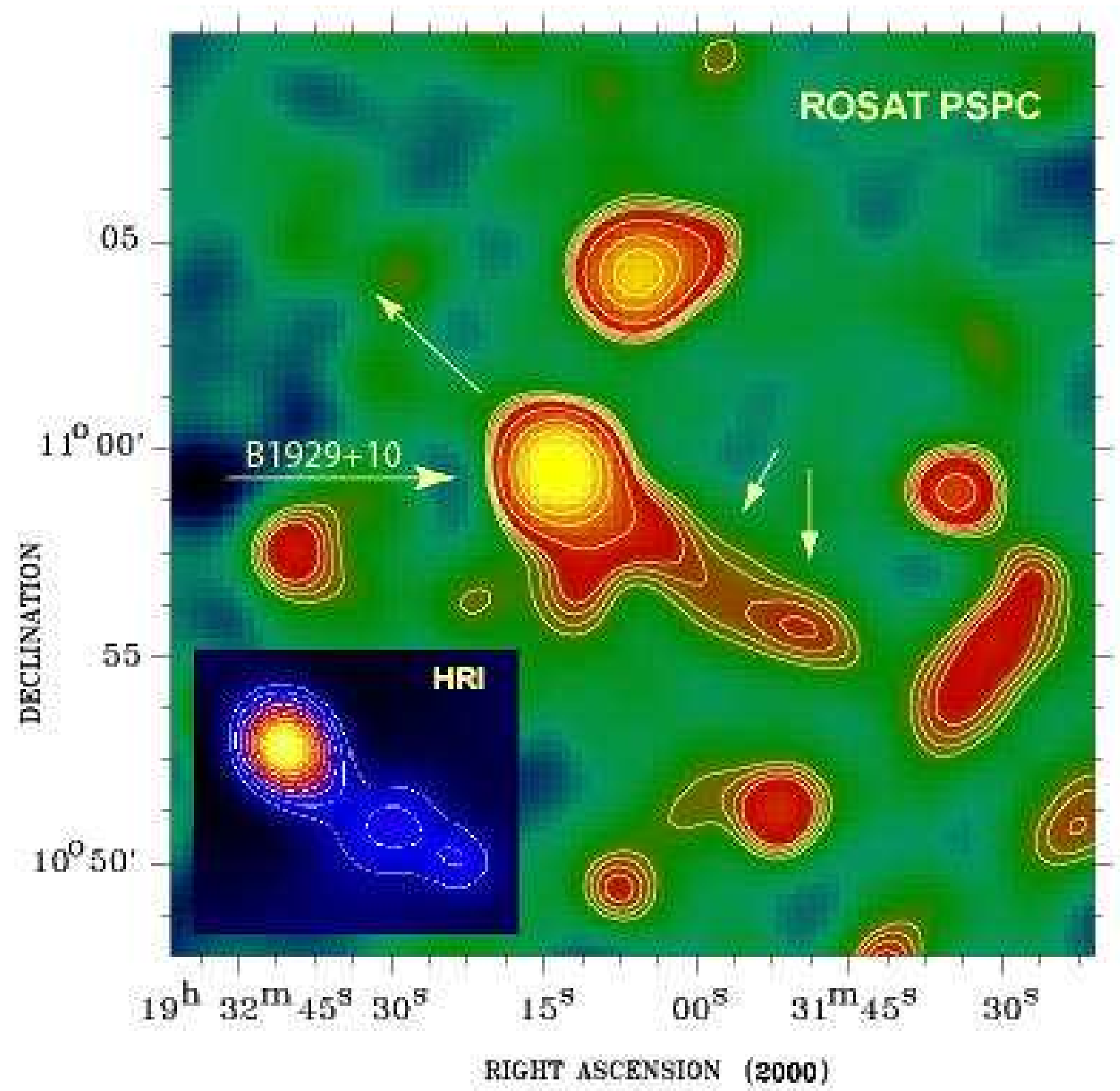

Fig. 2.- ROSAT PSPC's view of the sky region around PSR B1929+10 as seen in the $0.1-2.4 \mathrm{keV}$ energy band. The pulsar's proper motion direction and its X-ray trail are indicated by arrows. The trail length in this image is $\sim 9$ arcmin. The image has been smoothed with a $3 \sigma$ Gaussian filter. Contour lines are at the levels of $(0.98,1.02,1.07,1.11,1.33,1.77,2.22,2.66,3.11) \times 10^{-2} \mathrm{PSPC}$ counts/arcsec ${ }^{2}$. The inset in the lower left corner shows a zoomed view of the pulsar and a bright portion of its X-ray trail as reconstructed from the archival ROSAT HRI data using an adaptive kernel smoothing procedure. 


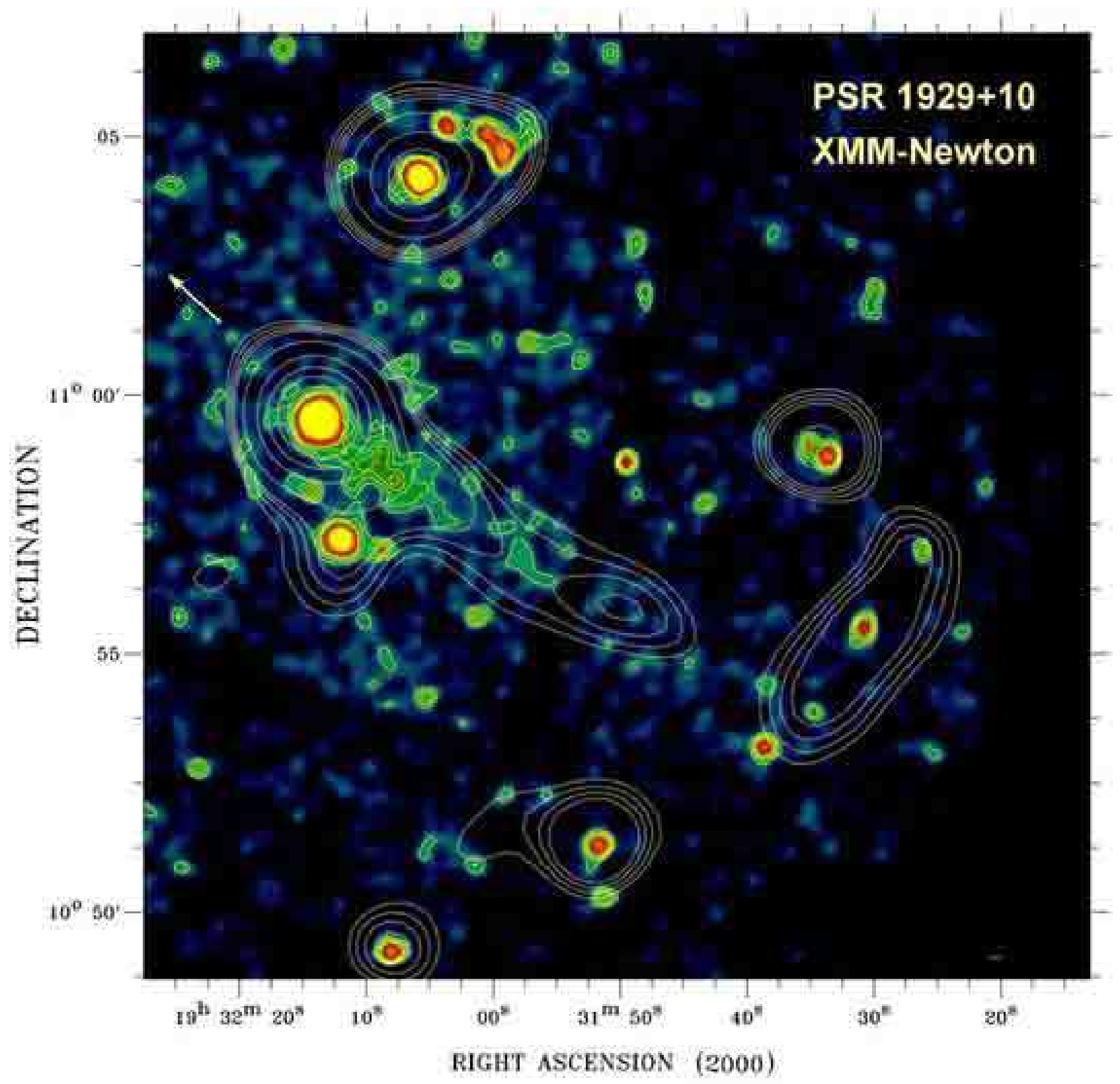

Fig. 3.- XMM-Newton's view of PSR B1929+10 and its environment as seen in the $0.2-$ $10 \mathrm{keV}$ energy band. Data from the EPIC-MOS1 and MOS2 detectors have been merged to produce the image. The image has been smoothed with a $3 \sigma$ Gaussian filter. Contour lines in the MOS1/2 image are at levels $(2.0,2.4,2.8,3.2) \times 10^{-2}$ EPIC-MOS1/2 counts/ $\operatorname{arcsec}^{2}$. Contour lines from the ROSAT PSPC image (cf. Figure 2) are overlaid with a $50 \%$ reduced opacity for comparison. The pulsar's proper motion direction is indicated by an arrow. 


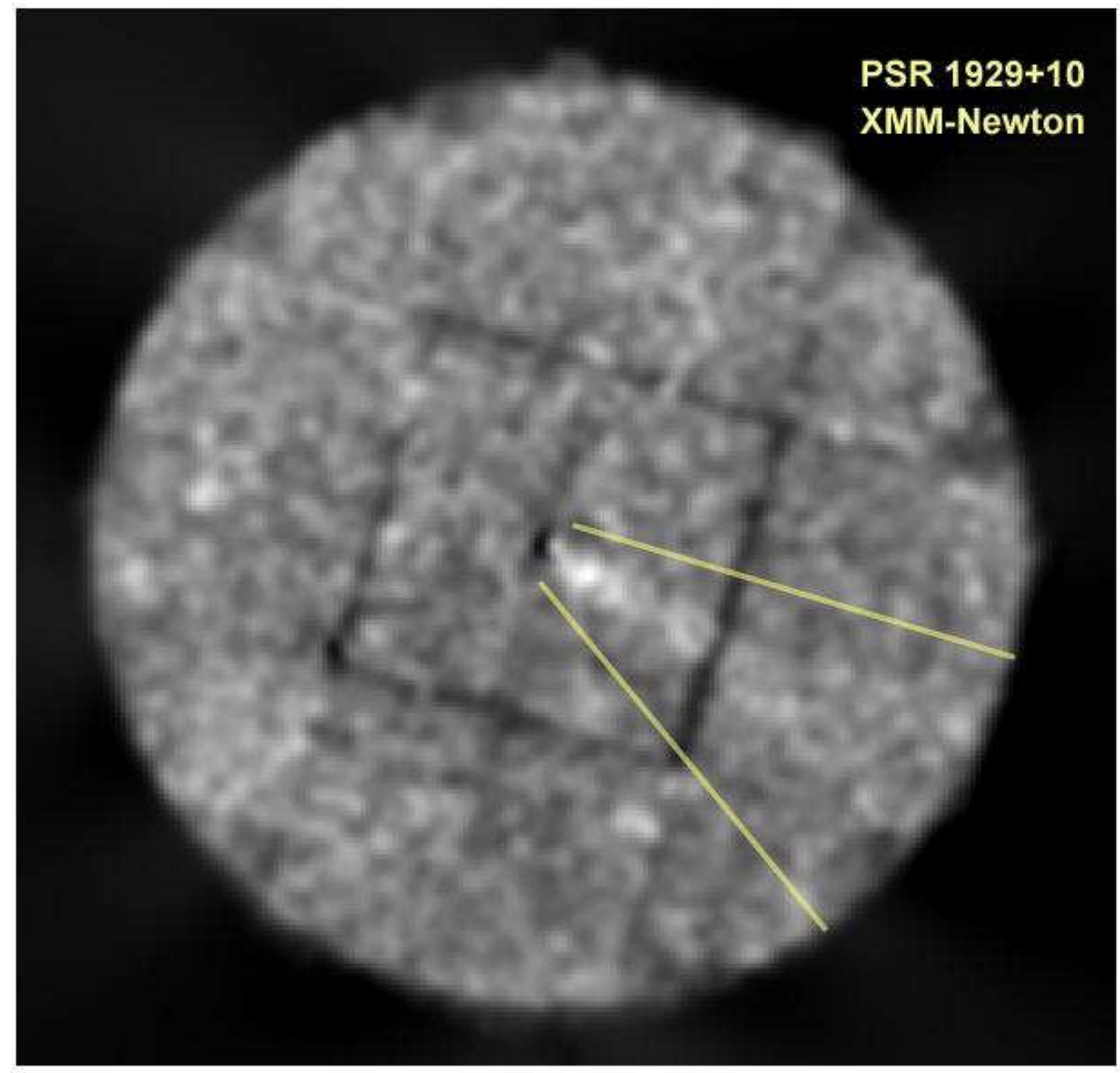

Fig. 4. - XMM-Newton MOS1/2 full 15 arcmin field of view of the sky region around PSR B1929+10as seen in the 0.2 - $10 \mathrm{keV}$ energy band. Applying corrections for vignetting and removing the X-ray point source contribution from the image the diffuse X-ray emission from the pulsar's trail becomes visible down to the edge of the MOS1/2 detectors. The segment of interest is indicated. 


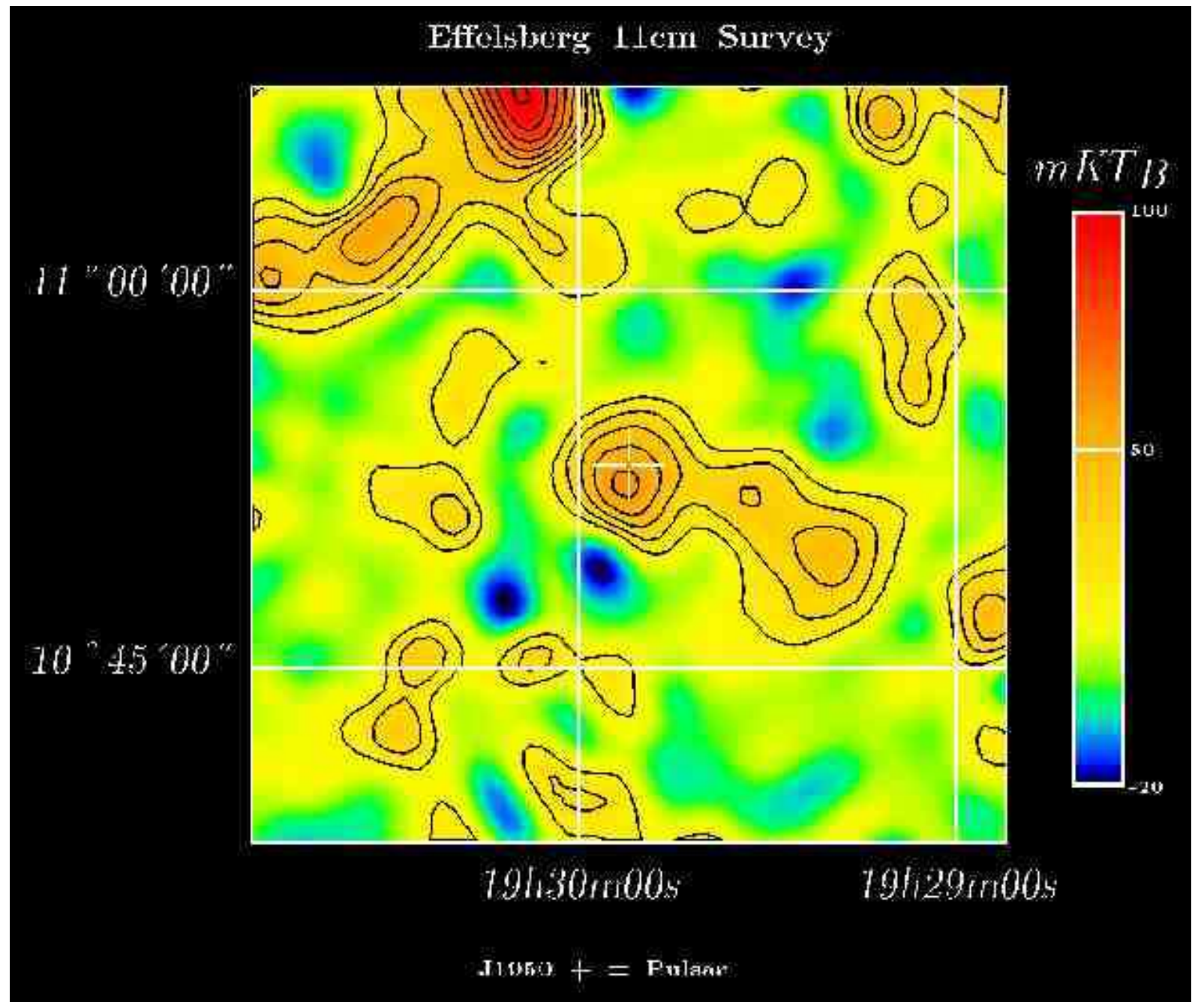

Fig. 5. - A $30 \times 30$ arcmin section of the $11 \mathrm{~cm}$ radio map around PSR B1929+10 as seen with the Effelsberg $100 \mathrm{~m}$ Radio Telescope. The map is centered on the pulsar. The smooth galactic background has been removed and the image was enhanced by unsharp masking on a scale of 7.5 arcmin. Beamsize (hpbw) was 4.3 arcmin and the rms $50 \mathrm{mJy}$. The length and the structure of the trial at $11 \mathrm{~cm}$ is comparable with what is observed from it in the X-ray band. The pulsar itself is seen as a polarized source with a flux of $200-250 \mathrm{mJy}$. Polarization of the other features was not detectable at the sensitivity of the survey. 


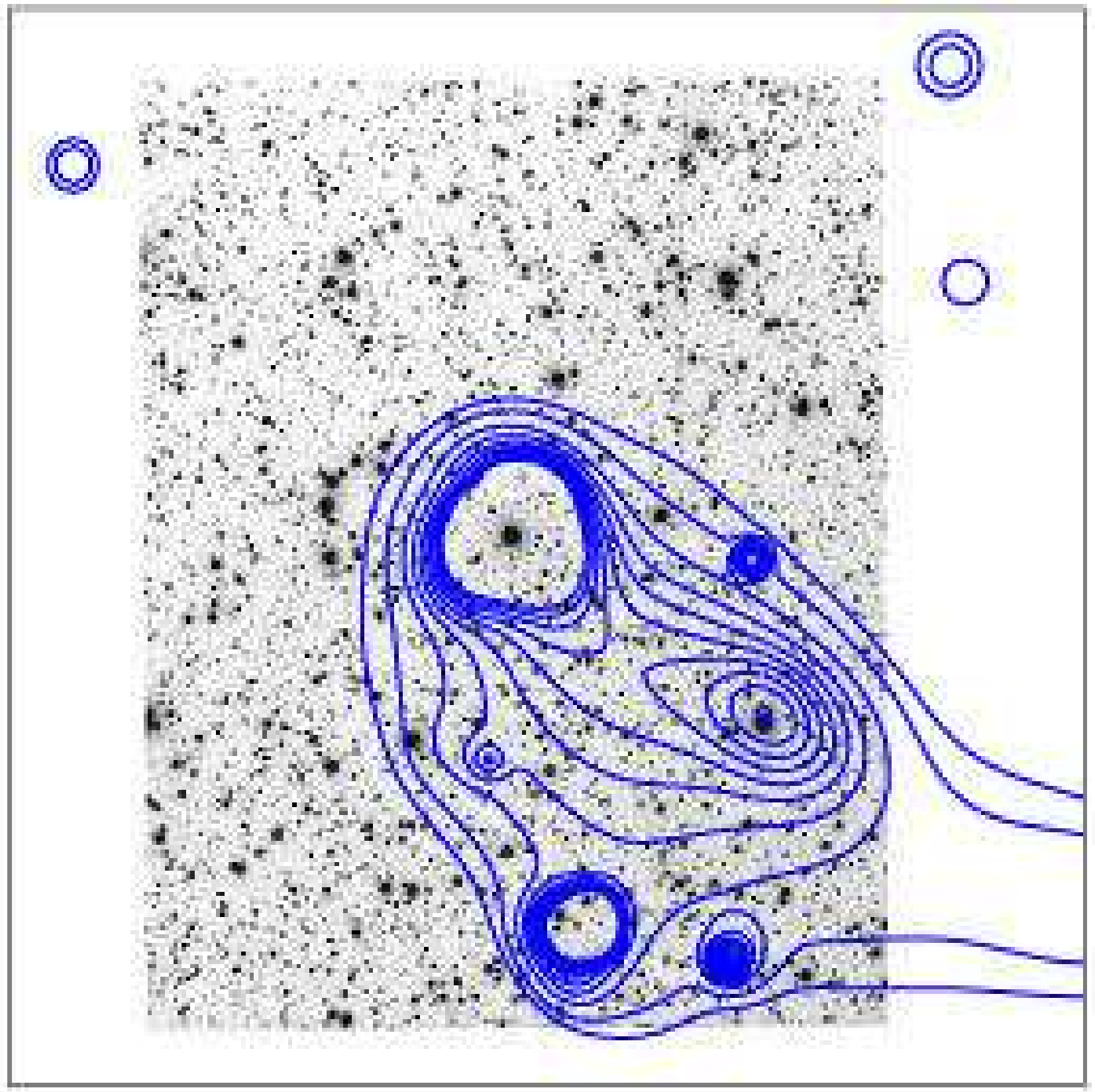

Fig. 6. - The $4.25 \times 5.41$ arcmin sky field around PSR B1929+10 as seen in in the light of $\mathrm{H}_{\alpha}$ with the ESO NTT. Overlaid are the contour lines from the XMM-Newton MOS1/2 image to which adaptive kernel smoothing was applied. 


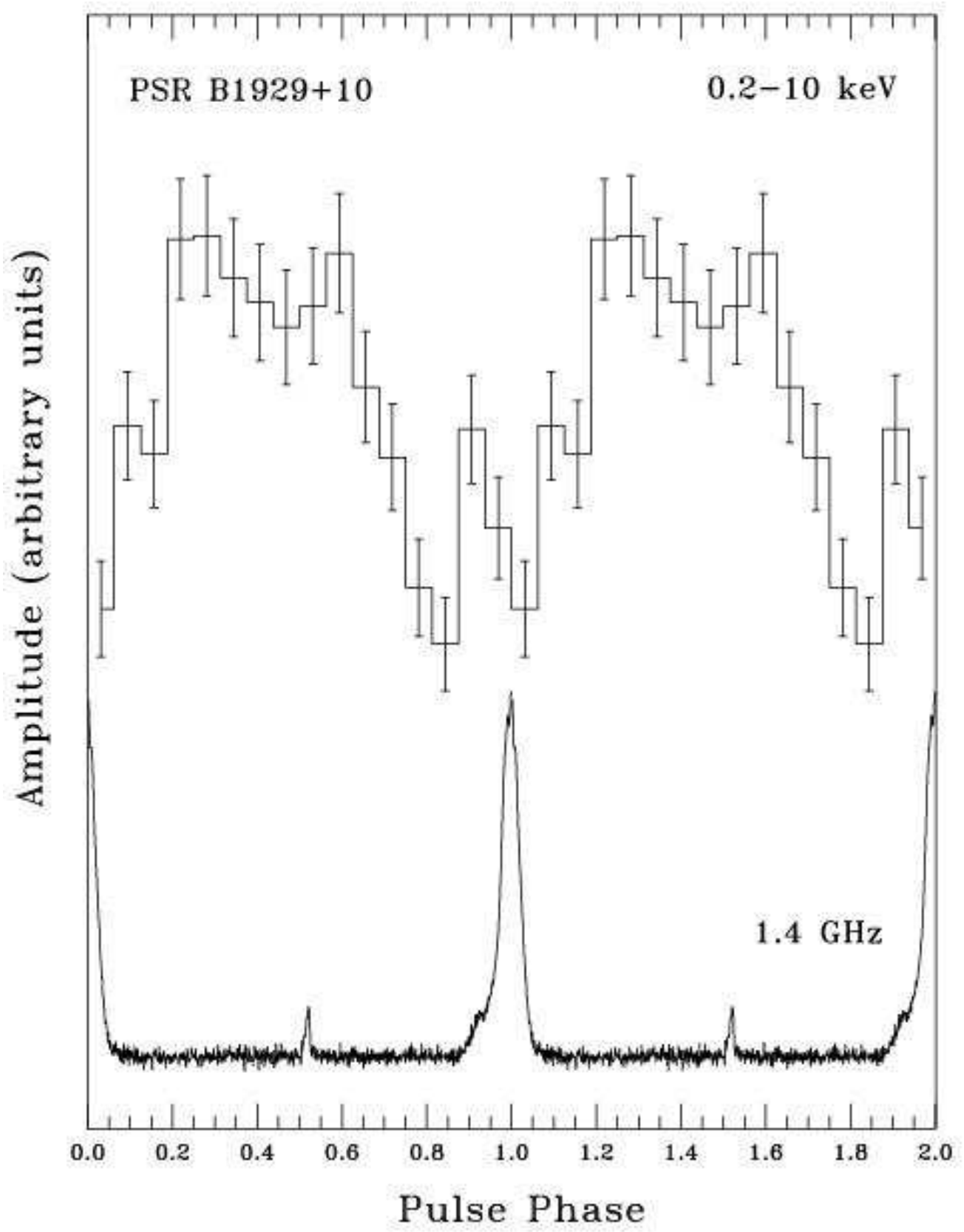

Fig. 7.- Integrated pulse profiles of PSR B1929+10 as observed in the $0.2-10 \mathrm{keV}$ band with the EPIC-PN Camera (top) and at $1.4 \mathrm{GHz}$ with the Jodrell Bank radio observatory (bottom). The radio profile is plotted on a logarithmic scale to better visualize the weak interpulse which has a displacement of $\sim 180^{\circ}$ from the main radio pulse peak. X-ray and radio profiles are phase related. Phase zero corresponds to $\mathrm{MJD}=53120.01448082337447$ (TDB at SSB). Two phase cycles are shown for clarity. 


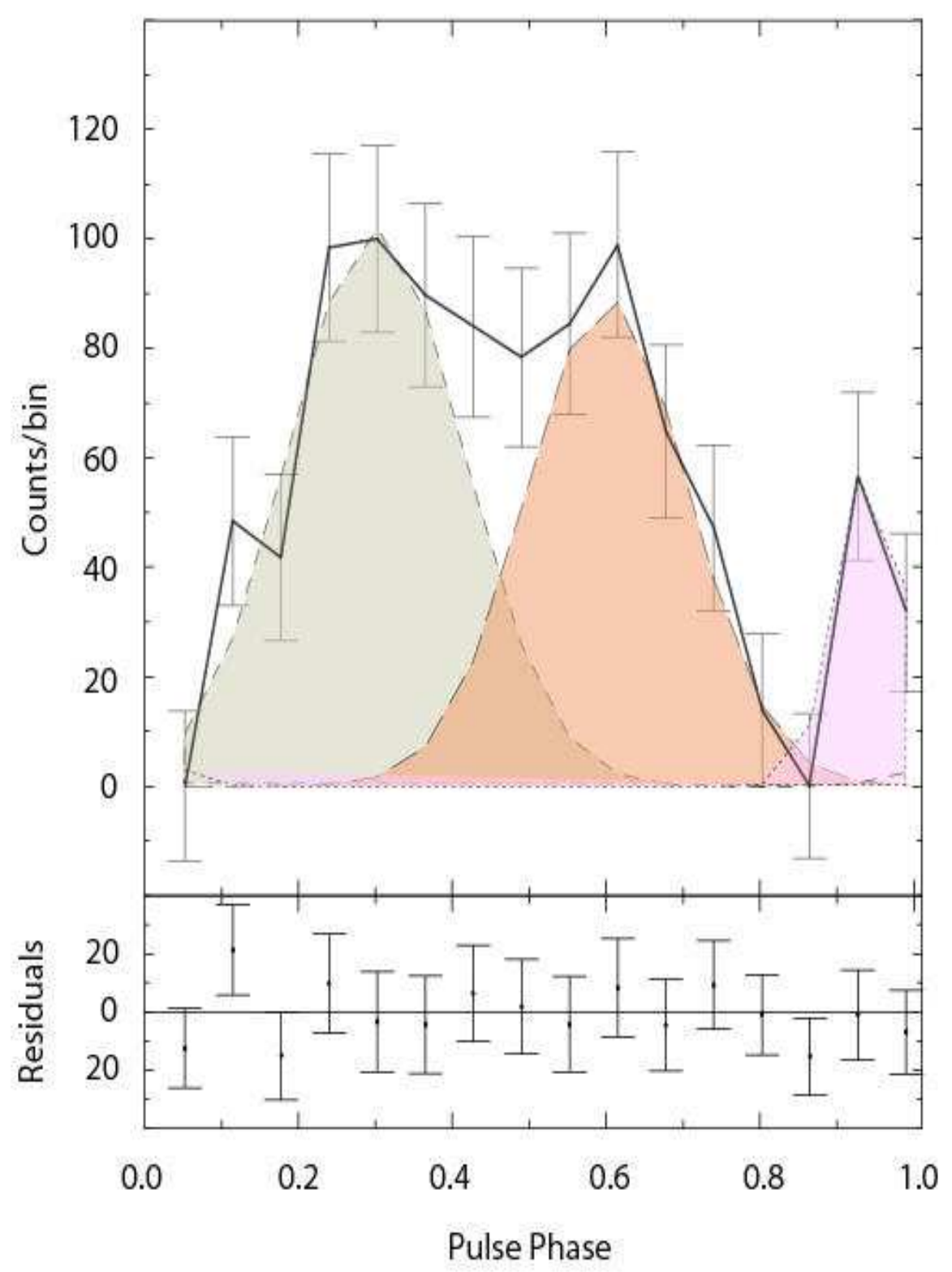

Fig. 8. - Results of fitting three Gaussians to the pulse profile of PSR B1929+10 observed in the $0.2-10 \mathrm{keV}$ energy band. The fit residuals in units of counts/bin are given in the lower panel. 


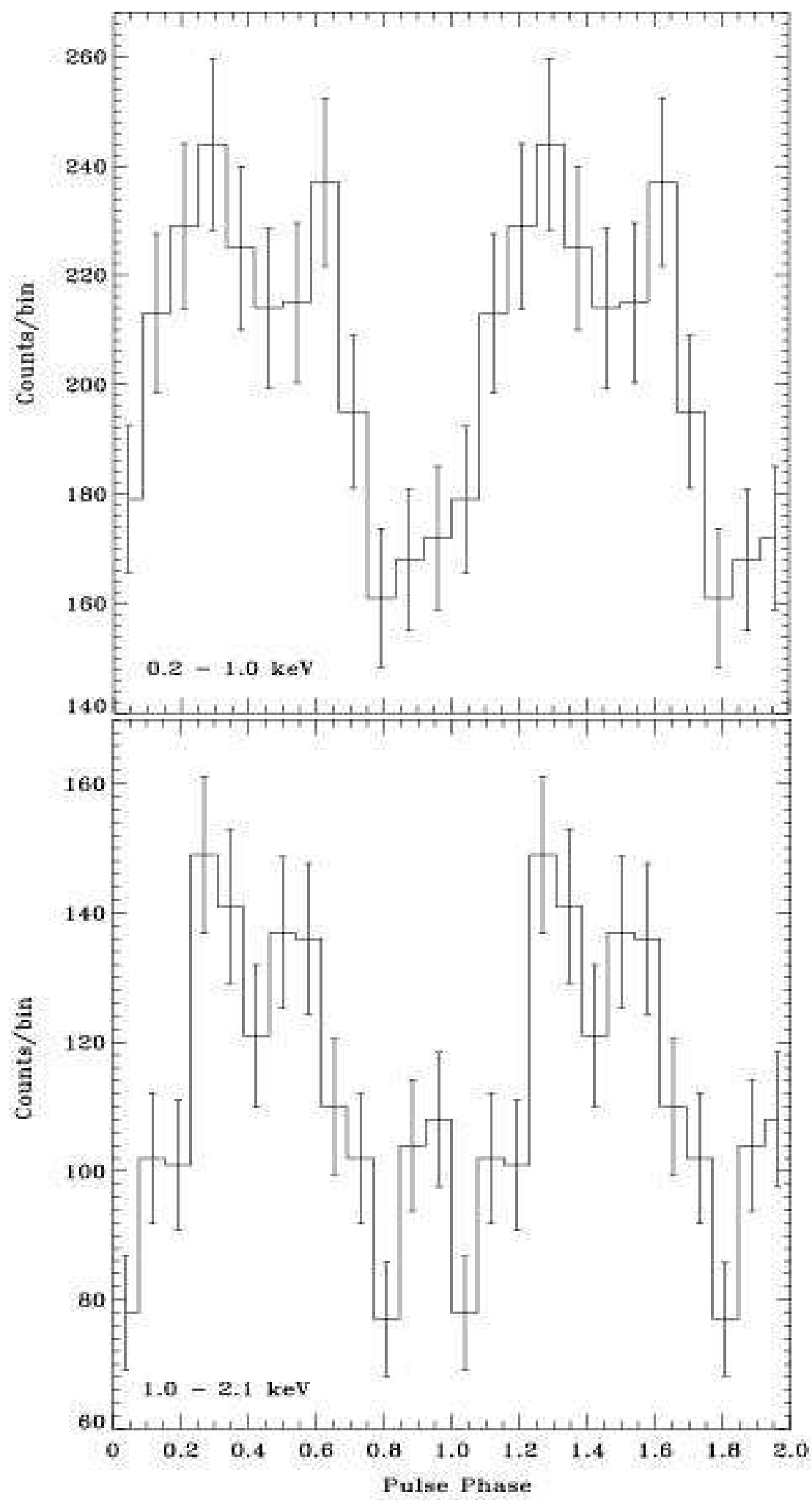

Fig. 9.- Integrated pulse profiles of PSR B1929+10 as observed in the energy bands $0.2-1.0$ $\mathrm{keV}$ and $1.0-2.1 \mathrm{keV}$ by the EPIC-PN camera. A narrow pulse component appears beyond $\sim 1 \mathrm{keV}$ near to phase 0 (1). Phase zero corresponds to MJD=53120.01448082337447 (TDB at SSB) and is the location of the radio main pulse peak. Two phase cycles are shown for clarity. 


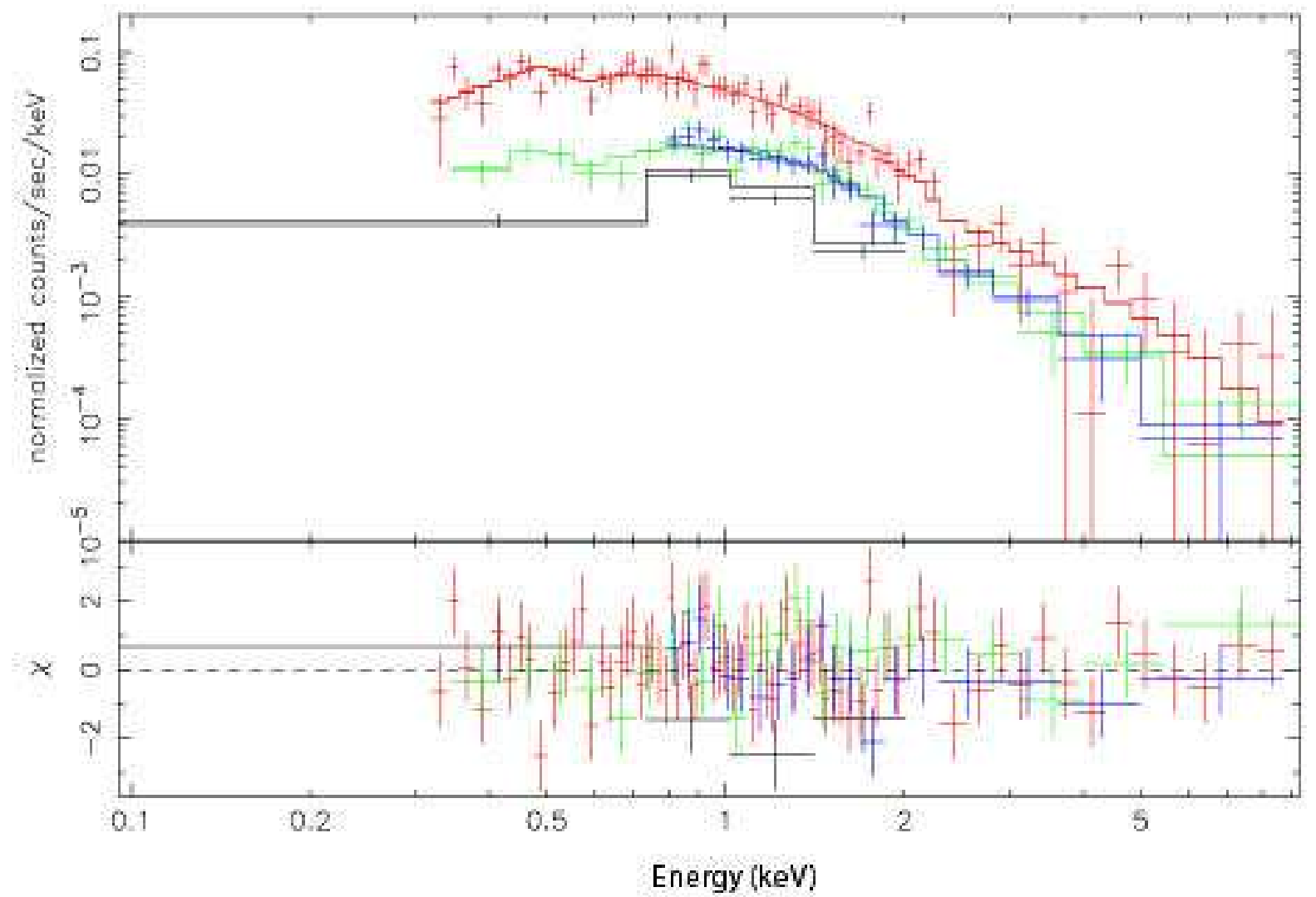

Fig. 10.- Energy spectrum of PSR B1929+10 as observed with the EPIC-PN (upper spectrum), the MOS1/2 detectors (middle spectra), and the ROSAT PSPC (lower spectrum) and simultaneously fitted to an absorbed power law model (upper panel) and contribution to the $\chi^{2}$ fit statistic (lower panel). 


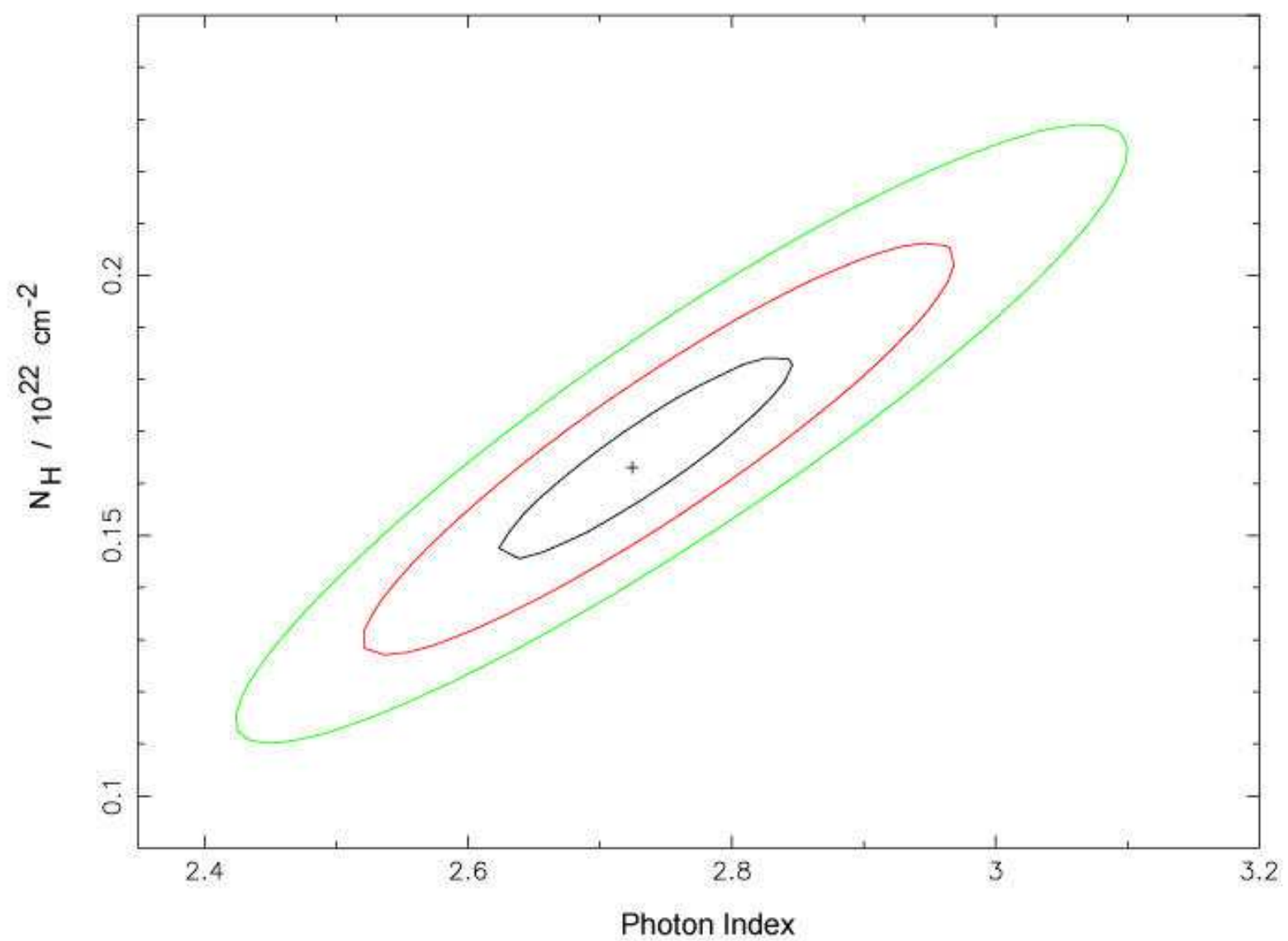

Fig. 11.- Contour plot showing the relative parameter dependence of the photon index vs. column absorption for the power law fit to the PSR B1929+10 XMM-Newton and ROSAT data. The three contours represent the $1 \sigma, 2 \sigma$ and $3 \sigma$ confidence contours for one parameter of interest. The '+' sign marks the best fit position, corresponding to $\chi_{\min }^{2}=0.989$ for 121 dof. 


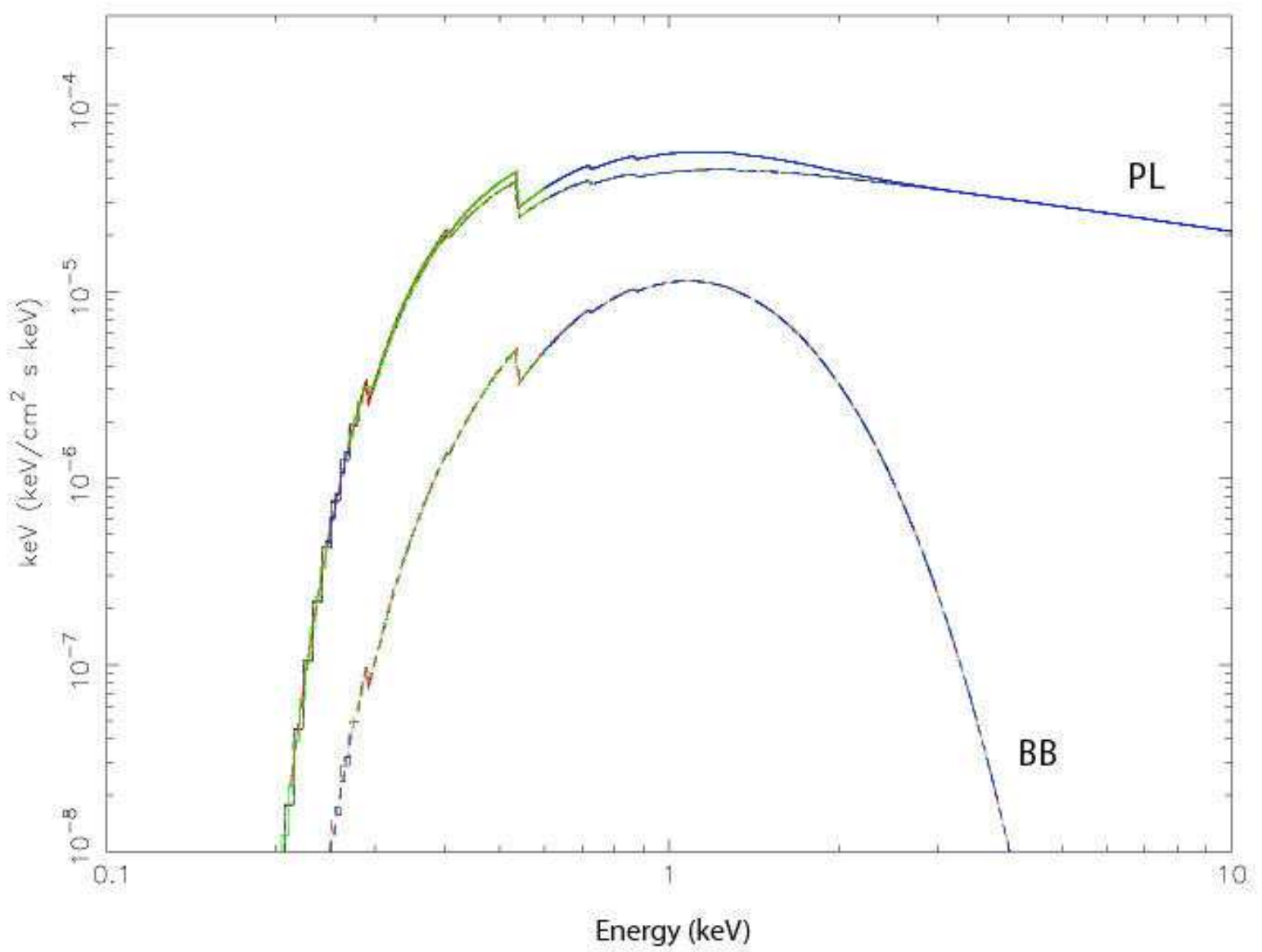

Fig. 12.- Blackbody plus power law spectral components and combined model as fitted to the spectral data of PSR B1929+10. 


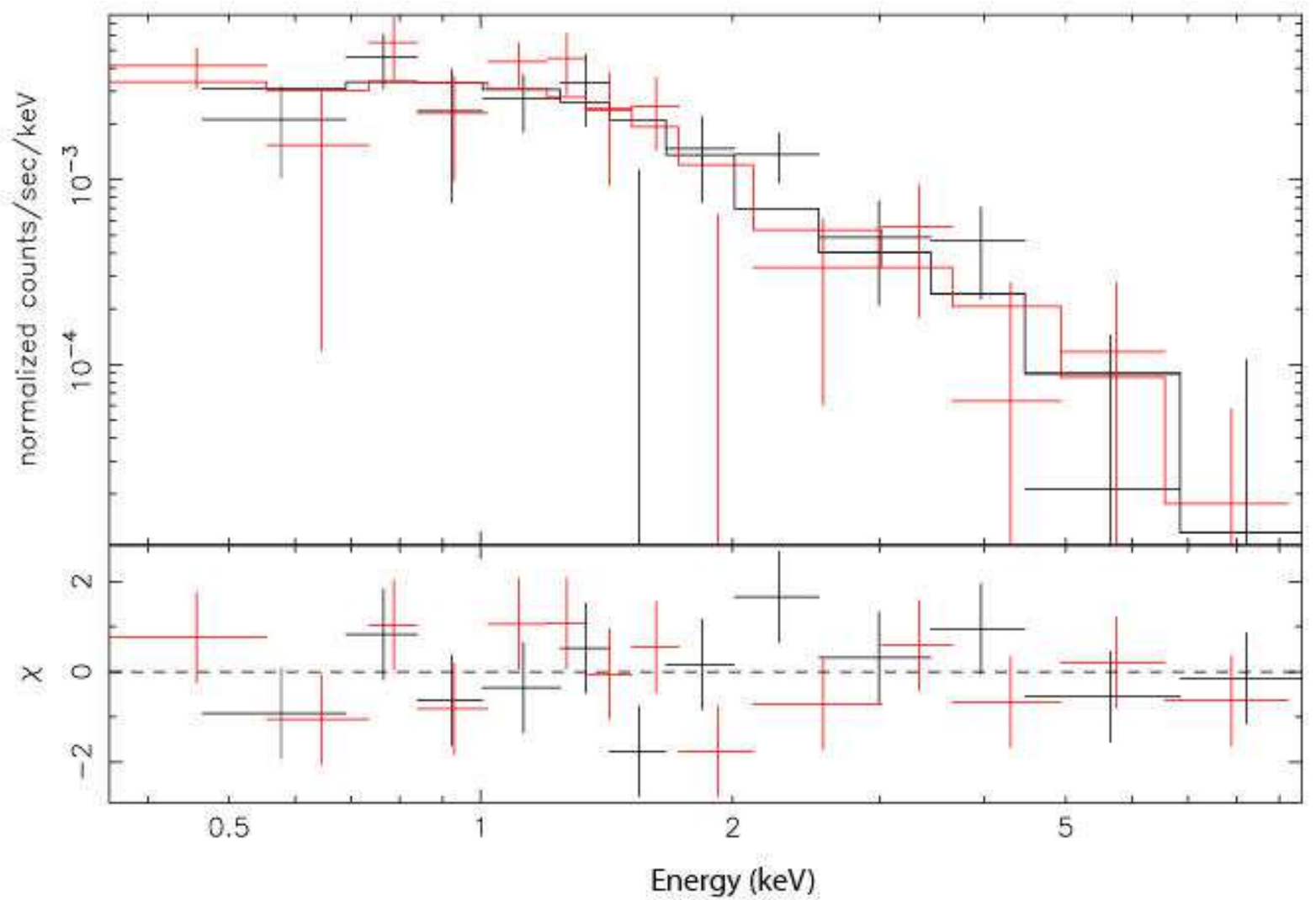

Fig. 13. - Energy spectrum of PSR B1929+10's X-ray trail observed with the EPIC-MOS1/2 detectors and simultaneously fitted to an absorbed power law model (upper panel) and contribution to the $\chi^{2}$ fit statistic (lower panel). 


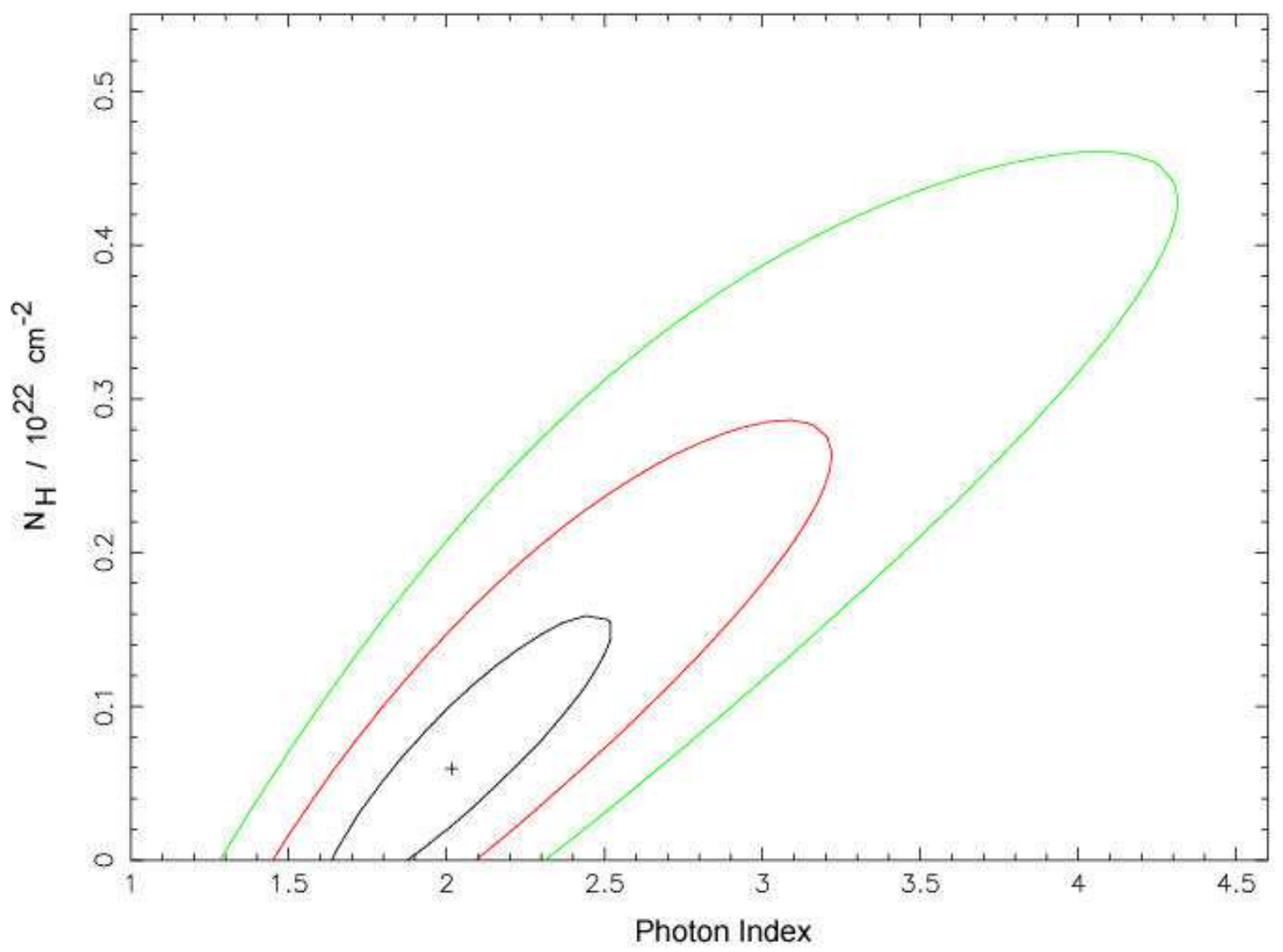

Fig. 14.- Contour plot showing the relative parameter dependence of the photon index vs. column absorption for the power law fit to the energy spectrum of PSR B1929+10's Xray trail. The three contours represent the $1 \sigma, 2 \sigma$ and $3 \sigma$ confidence levels for one parameter of interest. The ' + ' sign marks the best fit position, corresponding to $\chi_{\min }^{2}=0.885$ for 23 dof. 


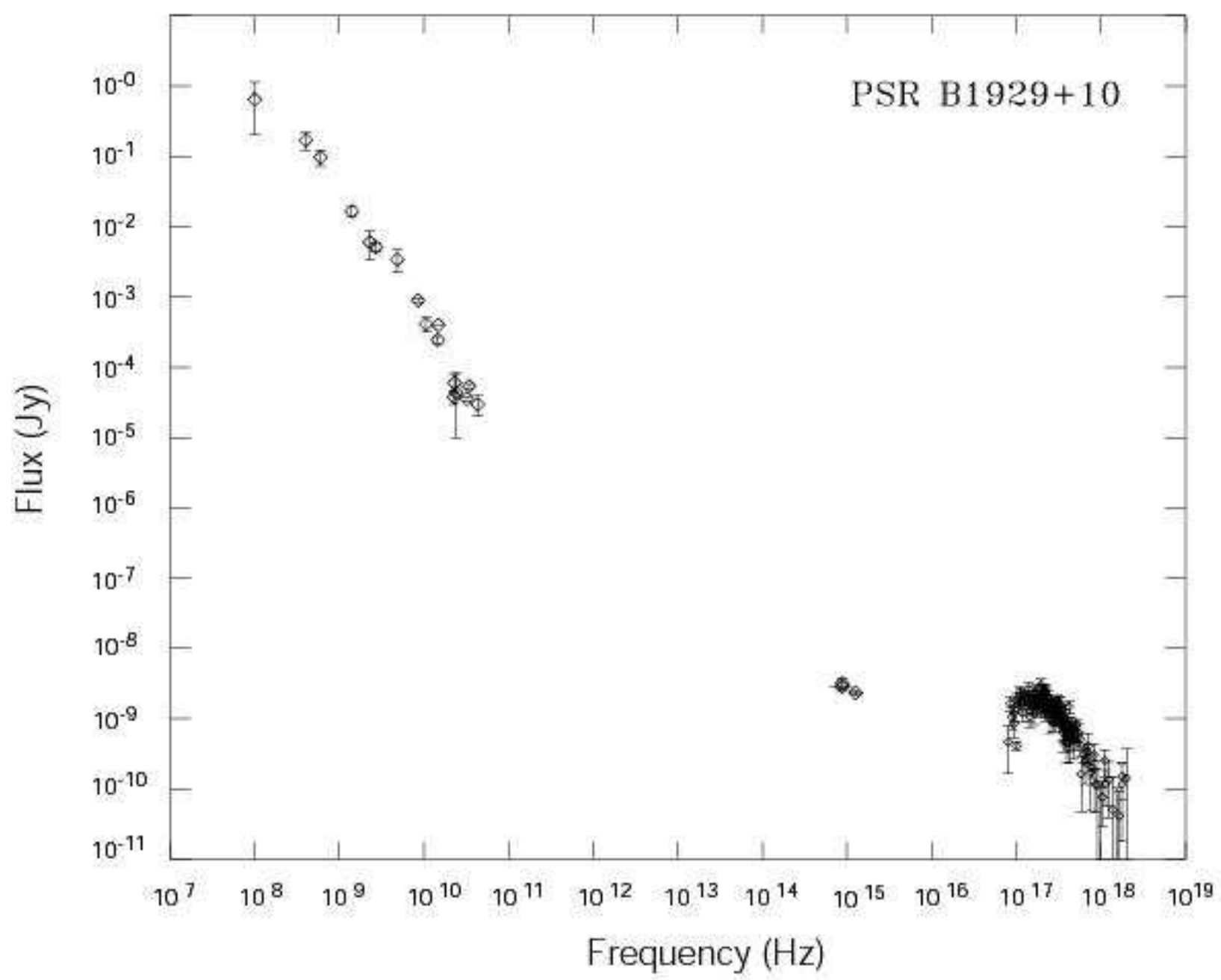

Fig. 15.- Combined radio, optical and X-ray spectral data of PSR B1929+10. To describe the optical and X-ray spectrum in terms of magnetospheric emission a broken power law spectral model with a break point fitted at $E_{\text {break }}=0.83_{-0.03}^{+0.05} \mathrm{keV}$ is required. 


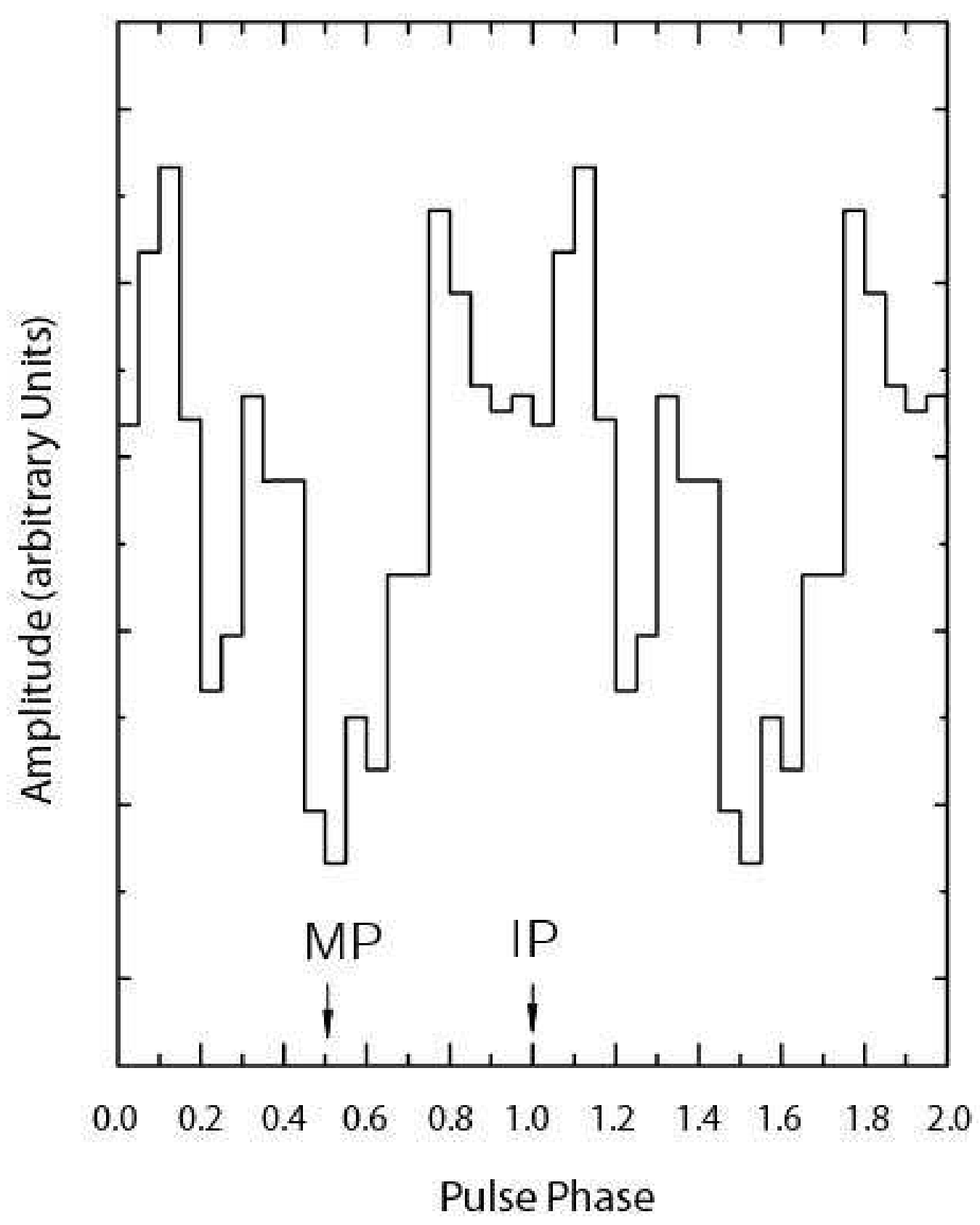

Fig. 16.- Simulated X-ray light curve of PSR B1929+10. Phase zero refers to the plane containing both the magnetic and rotational axes, and the related main pulse (MP) and interpulse (IP) of the radio profile are indicated by arrows. Two phase cycles are shown. 